TRANSACTIONS OF THE

AMERICAN MATHEMATICAL SOCIETY

Volume 362, Number 11, November 2010, Pages 6079-6103

S 0002-9947(2010)05067-6

Article electronically published on June 16, 2010

\title{
NORMAL AUTOMORPHISMS OF RELATIVELY HYPERBOLIC GROUPS
}

\author{
A. MINASYAN AND D. OSIN
}

Dedicated to Professor A.L. Shmelkin on the occasion of his 70th birthday.

\begin{abstract}
An automorphism $\alpha$ of a group $G$ is normal if it fixes every normal subgroup of $G$ setwise. We give an algebraic description of normal automorphisms of relatively hyperbolic groups. In particular, we show that for any such group $G, \operatorname{Inn}(G)$ has finite index in the subgroup $A_{u t}(G)$ of normal automorphisms. If, in addition, $G$ is non-elementary and has no finite non-trivial normal subgroups, then $\operatorname{Aut}_{n}(G)=\operatorname{Inn}(G)$. As an application, we show that $\operatorname{Out}(G)$ is residually finite for every finitely generated residually finite group $G$ with infinitely many ends.
\end{abstract}

\section{Contents}

1. Introduction

2. Preliminaries

3. Special elements in relatively hyperbolic groups 6086

4. Technical lemmas 6088

5. Commensurating automorphisms of relatively hyperbolic groups 6093

6. Group-theoretic Dehn surgery and normal automorphisms 6096

7. Free products and groups with infinitely many ends 6099

Acknowledgments $\quad 6101$

References 6101

\section{Introduction}

Recall that an automorphism $\alpha \in \operatorname{Aut}(G)$ of a group $G$ is said to be normal if $\alpha(N)=N$ for every normal subgroup $N$ of $G$. The subset of normal automorphisms, denoted by $\operatorname{Aut}_{n}(G)$, is clearly a subgroup of $A u t(G)$. Obviously every inner automorphism is normal. Throughout this paper we denote by $O u t_{n}(G)$ the quotient group $\operatorname{Aut}_{n}(G) / \operatorname{Inn}(G)$.

Received by the editors September 30, 2008 and, in revised form, March 25, 2009 and March 31, 2009.

2010 Mathematics Subject Classification. Primary 20F65, 20F67, 20 E26.

Key words and phrases. Relatively hyperbolic group, normal automorphism, outer automorphism group, group with infinitely many ends, residual finiteness, group-theoretic Dehn surgery.

The first author was supported by the Swiss National Science Foundation grant PP002-116899.

The second author was supported by the NSF grant DMS-0605093 and by the RFBR grant 05-01-00895.

(c)2010 American Mathematical Society
Reverts to public domain 28 years from publication 6079 
The study of normal automorphisms originates from the result of Lubotzky stating that $\operatorname{Out}_{n}(G)$ is trivial for any non-abelian free group [25. Since then similar results have been proved for many other classes of groups. For example, Out $_{n}(G)=\{1\}$ for non-trivial free products [29], fundamental groups of closed surfaces of negative Euler characteristic [6], non-abelian free Burnside groups of large odd exponent [9], non-abelian free solvable groups [37, and free nilpotent groups of class $c=2$ (for $c \geq 3$ this is not true) [13. On the other hand, every group can be realized as $\operatorname{Out}(G)$ for a suitable simple group $G[12$. Since every automorphism of a simple group is normal, every group appears as $O u t_{n}(G)$ for some $G$. Furthermore, every countable group can be realized as $\operatorname{Out}_{n}(G)$ for some finitely generated group $G$ [27, 30].

The main goal of this paper is to study normal automorphisms of relatively hyperbolic groups. The notion of a relatively hyperbolic group was originally suggested by Gromov [16] and has been elaborated upon in many papers since then [7, 11, 14, 22, 33, 44]. The class of relatively hyperbolic groups includes (ordinary) hyperbolic groups, fundamental groups of finite-volume complete Riemannian manifolds of pinched negative curvature [7, 14, groups acting freely on $\mathbb{R}^{n}$-trees [20] (in particular, limit groups arising in the solutions of the Tarski problem [24, 40]), non-trivial free products and their small cancellation quotients 33, groups acting geometrically on $C A T(0)$ spaces with isolated flats [23, and many other examples.

In this paper we neither assume relatively hyperbolic groups to be finitely generated nor the collection of peripheral subgroups to be finite. (The reader is referred to the next section for the precise definition.) However we do assume that all peripheral subgroups are proper to exclude the case of a group hyperbolic relative to itself. Further on, we will say that a group $G$ is non-elementary if it is not virtually cyclic.

In general $\mathrm{Out}_{n}(G)$ is not necessarily trivial even for ordinary hyperbolic groups. Indeed, it is known (see [38]) that certain finite groups in $L$ possess non-inner automorphisms which map every element to its conjugate. One can therefore construct many hyperbolic groups $G$ with non-trivial $O u t_{n}(G)$ by taking any hyperbolic group $H$ and considering the direct product $G=H \times L$. The first result of our paper shows that non-trivial finite normal subgroups are essentially the only sources of non-inner normal automorphisms.

More precisely, every relatively hyperbolic group $G$ contains a unique maximal finite normal subgroup (see Corollary 2.6). We denote it by $E(G)$. Further let $C(G)$ denote the centralizer of $E(G)$ in $G$.

Theorem 1.1. Suppose that $G$ is a non-elementary relatively hyperbolic group and let $\alpha \in \operatorname{Aut}_{n}(G)$. Then there exist an element $w \in G$ and a set map $\varepsilon: G \rightarrow E(G)$ such that $\varepsilon(C(G))=\{1\}$ and $\alpha(g)=w g \varepsilon(g) w^{-1}$ for every $g \in G$.

In fact, Theorem 1.1 is a particular case of a more general result about normal automorphisms of subgroups of relatively hyperbolic groups (see Theorem6.4). The corollary below follows easily from Theorem 1.1 and the observation that $C(G)$ has finite index in $G$, being the centralizer of a finite normal subgroup.

Corollary 1.2. Suppose that $G$ is a relatively hyperbolic group. Then the following hold.

(a) Out $_{n}(G)$ is finite. 
(b) If $G$ is non-cyclic and contains no non-trivial finite normal subgroups, then $\mathrm{Out}_{n}(G)=\{1\}$.

This corollary generalizes the results about free groups [25], free products [29], and surface groups [6] cited above. It also implies the result of Metaftsis and Sykiotis [26] stating that for every non-elementary finitely generated relatively hyperbolic group $G, \operatorname{Inn}(G)$ has finite index in the group $A u t_{c}(G)$ of pointwise inner automorphisms of $G$. Recall that an automorphism of $G$ is pointwise inner if it preserves conjugacy classes. Clearly $A u t_{c}(G) \leq A u t_{n}(G)$. Thus finiteness of $O u t_{n}(G)$ implies that of $A_{u t}(G) / \operatorname{Inn}(G)$. The converse is not true in general. For instance, if $G$ is free nilpotent of class at least 3 , we have $A u t_{c}(G)=\operatorname{Inn}(G)$ while $\left|O u t_{n}(G)\right|=\infty$ 13 .

It is also worth noting that our methods are quite different from those of 26 . Indeed we use the group-theoretic version of Dehn surgery introduced in [18, 19, 32 and 'component analysis' developed in 33, 27, while Metaftsis and Sykiotis employed the Bestvina-Paulin approach [5, 34] based on ultralimits and group actions on $\mathbb{R}$-trees.

In order to prove Theorem 1.1] we introduce a new subclass of automorphisms of any given group and investigate it in the case of relatively hyperbolic groups.

Definition 1.3. Let $G$ be a group. We say that an automorphism $\varphi \in A u t(G)$ is commensurating if for every $g \in G$ there exist $h \in G$ and $m, n \in \mathbb{Z} \backslash\{0\}$ such that $(\varphi(g))^{n}=h g^{m} h^{-1}$. In other words, $\varphi$ is commensurating if for each $g \in G, \varphi(g)$ is commensurable to $g$ in $G$ (see Definition 4.1).

It is clear that the subset $A u t_{c o m m}(G)$ of commensurating automorphisms of $G$ forms a subgroup of $A u t(G)$ and $\operatorname{Inn}(G) \leq A u t_{c}(G) \leq A u t_{\text {comm }}(G)$.

In Section 5 we study commensurating automorphisms of relatively hyperbolic groups and obtain a complete description of them:

Corollary 1.4. Let $G$ be a non-elementary relatively hyperbolic group and $\varphi \in$ Aut $(G)$. The following conditions are equivalent:

(i) $\varphi$ is commensurating;

(ii) there is a set map $\varepsilon: G \rightarrow E(G)$, whose restriction to $C(G)$ is a homomorphism, and an element $w \in G$ such that for every $g \in G, \varphi(g)=$ $w(g \varepsilon(g)) w^{-1}$.

In particular, if $E(G)=\{1\}$, then every commensurating automorphism of $G$ is inner.

In Section 6] using the algebraic version of Dehn filling, we show that each normal automorphism of a relatively hyperbolic group must be commensurating. After this, Theorem 1.1 follows quite quickly from the above description of commensurating automorphisms.

Our methods can also be used to prove residual finiteness of some outer automorphism groups. A well-known theorem of Baumslag states that the automorphism group of a finitely generated residually finite group is residually finite [4. In general, the analogous property does not hold for the group of outer automorphisms. Indeed, Bumagina and Wise showed that every finitely presented group is realized as $\operatorname{Out}(G)$ for a suitable finitely generated residually finite group $G$ [8]. However we prove that Baumslag's theorem does have an 'outer' analogue for groups with 
infinitely many ends. We refer to 41 for the geometric definition of ends and recall that a finitely generated group has infinitely many ends if and only if it splits non-trivially as an amalgamated free product $A *_{S} B$ or an $H N N$-extension $A *_{S}$ over a finite group $S$ [41, 42.

Theorem 1.5. Let $G$ be a finitely generated residually finite group with infinitely many ends. Then $\operatorname{Out}(G)$ is residually finite.

Note that the condition demanding residual finiteness of $G$ cannot be removed. Indeed, if $H$ is any finitely generated group that has trivial center and is not residually finite, then the group $G=H * \mathbb{Z}$ has infinitely many ends and $H$ is embedded into $\operatorname{Out}(G)$ ( $H$ acts on itself by conjugation and trivially on $\mathbb{Z}$, which gives rise to an action of $H$ by outer automorphisms on the free product $H * \mathbb{Z}=G$ ). Thus $\operatorname{Out}(G)$ is not residually finite.

The standard way of proving residual finiteness of $\operatorname{Out}(G)$ is based on the following result of Grossman [17]: if a group $G$ is finitely generated and conjugacy separable, then $\operatorname{Aut}(G) / \operatorname{Aut}_{c}(G)$ is residually finite. In particular, Out $(G)$ is residually finite whenever $G$ is finitely generated, conjugacy separable, and $A u t_{c}(G)=\operatorname{Inn}(G)$. Recall that a group $G$ is said to be conjugacy separable if for any two non-conjugate elements $g, h \in G$ there exists a homomorphism $\varepsilon: G \rightarrow K$, where $K$ is finite, such that $\varepsilon(g)$ and $\varepsilon(h)$ are not conjugate in $K$.

This approach has been successfully used to prove residual finiteness of $\operatorname{Out}(G)$, where $G$ is a free group of finite rank [17, the fundamental group of a closed surface [17, 2], the fundamental group of a Seifert manifold with non-trivial boundary [1, etc. If $G$ is a finitely generated conjugacy separable non-elementary relatively hyperbolic group, the above-mentioned result from [26 implies that every virtually torsion-free subgroup of $\operatorname{Out}(G)$ is residually finite [26, Theorem 1.1].

However there is no hope to use Grossman's idea to prove Theorem 1.5 since we only assume the group $G$ to be residually finite, which is much weaker than conjugacy separability. Indeed there are many examples of finitely generated residually finite groups that are not conjugacy separable (e.g., the group of unimodular matrices $G L(n, \mathbb{Z})$ for $n \geq 3$; see 36 ). To construct such an example with infinitely many ends, we can simply take $G=H * \mathbb{Z}$, where $H$ is finitely generated, residually finite, but not conjugacy separable. It is easy to show that $G$ will also be finitely generated, residually finite, but not conjugacy separable.

Our approach is different and is based on the following observation. Let $A u t_{n}^{f}(G)$ denote the group of automorphisms of $G$ that stabilize every normal subgroup of finite index (setwise). Then $\operatorname{Aut}(G) / \operatorname{Aut}_{n}^{f}(G)$ is residually finite for every finitely generated group $G$. The following result plays the crucial role in the proof of Theorem 1.5. It also seems to be of independent interest. Its proof essentially uses the fact that free products are hyperbolic relative to their free factors, which allows us to employ the techniques developed in the proof of Theorem 1.1.

Theorem 1.6. Suppose that $G=A * B$, where $A, B$ are non-trivial residually finite groups. Then $\operatorname{Aut}_{n}^{f}(G)=\operatorname{Inn}(G)$.

\section{Preliminaries}

Notation. Given a group $G$ generated by a subset $S \subseteq G$, we denote by $\Gamma(G, S)$ the Cayley graph of $G$ with respect to $S$ and by $|g|_{S}$ the word length of an element $g \in G$. If $p$ is a (combinatorial) path in $\Gamma(G, S)$, then let $\operatorname{Lab}(p)$ denote its label, 
$\mathrm{L}(p)$ denote its length, and $p_{-}$and $p_{+}$denote its starting and ending vertex. The notation $p^{-1}$ will be used for the path in $\Gamma(G, S)$ obtained by traversing $p$ backwards. By saying that $o=p_{1} \ldots p_{k}$ is a cycle in $\Gamma(G, S)$ we will mean that $o$ is obtained as a consecutive concatenation of paths $p_{1}, \ldots, p_{k}$ such that $\left(p_{i+1}\right)_{-}=\left(p_{i}\right)_{+}$for $i=1, \ldots, k-1$ and $\left(p_{k}\right)_{+}=\left(p_{1}\right)_{-}$.

For a word $W$ written in the alphabet $S,\|W\|$ will denote its length. For two words $U$ and $V$ we shall write $U \equiv V$ to denote the letter-by-letter equality between them. The normal closure of a subset $K \subseteq G$ in a group $G$ (i.e., the minimal normal subgroup of $G$ containing $K)$ is denoted by $\langle\langle K\rangle\rangle^{G}$, or simply by $\langle\langle K\rangle$ if omitting $G$ does not lead to confusion. For any group elements $g$ and $t, g^{t}$ denotes $t^{-1} g t$. If $A \subseteq G$, then $A^{t}=\left\{a^{t} \mid a \in A\right\}$. For a subgroup $H \leq G, N_{G}(H)$ denotes the normalizer of an $H$ in $G$. That is, $N_{G}(H)=\left\{g \in G \mid g H g^{-1}=H\right\}$. Similarly by $C_{G}(H)$ we denote the centralizer of $H$ in $G$, that is,

$$
C_{G}(H)=\{g \in G \mid g h=h g, \forall h \in H\} .
$$

Finally for two subsets $A, B$ of $G$, their product is the subset $A B=\{a b \mid a \in A, b \in$ $B\}$.

Relatively hyperbolic groups. In this paper we use the notion of relative hyperbolicity, which is sometimes called strong relative hyperbolicity and goes back to Gromov [16. There are many equivalent definitions of (strongly) relatively hyperbolic groups [7, 11, 14, 33. We recall the isoperimetric characterization suggested in [33], which is most suitable for our purposes. That relative hyperbolicity in the sense of [7, 14, 16] implies relative hyperbolicity in the sense of Definition 2.1]stated below is essentially due to Rebbechi [35. (Indeed it was proved in 35] under the additional technical condition that the groups under consideration are finitely presented.) In the full generality, this implication and the converse one were proved in 33.

Let $G$ be a group, $\left\{H_{\lambda}\right\}_{\lambda \in \Lambda}$ - a collection of proper subgroups of $G, X$-a subset of $G$. We say that $X$ is a relative generating set of $G$ with respect to $\left\{H_{\lambda}\right\}_{\lambda \in \Lambda}$ if $G$ is generated by $X$ together with the union of all $H_{\lambda}$. (In what follows we always assume $X$ to be symmetric.) In this situation the group $G$ can be regarded as a quotient group of the free product

$$
F=\left(*_{\lambda \in \Lambda} H_{\lambda}\right) * F(X),
$$

where $F(X)$ is the free group with the basis $X$. If the kernel of the natural homomorphism $F \rightarrow G$ is the normal closure of a subset $\mathcal{R}$ in the group $F$, we say that $G$ has relative presentation

$$
\left\langle X, H_{\lambda}, \lambda \in \Lambda \mid \mathcal{R}\right\rangle .
$$

If $|X|<\infty$ and $|\mathcal{R}|<\infty$, the relative presentation (2) is said to be finite and the group $G$ is said to be finitely presented relative to the collection of subgroups $\left\{H_{\lambda}\right\}_{\lambda \in \Lambda}$.

Set

$$
\mathcal{H}=\bigsqcup_{\lambda \in \Lambda}\left(H_{\lambda} \backslash\{1\}\right)
$$


Given a word $W$ in the alphabet $X \cup \mathcal{H}$ such that $W$ represents 1 in $G$, there exists an expression

$$
W \stackrel{F}{=} \prod_{i=1}^{k} f_{i}^{-1} R_{i}^{ \pm 1} f_{i}
$$

with the equality in the group $F$, where $R_{i} \in \mathcal{R}$ and $f_{i} \in F$ for $i=1, \ldots, k$. The smallest possible number $k$ in a representation of the form (4) is called the relative area of $W$ and is denoted by $\operatorname{Area}^{\text {rel }}(W)$.

Definition 2.1 (33). A group $G$ is hyperbolic relative to a collection of proper subgroups $\left\{H_{\lambda}\right\}_{\lambda \in \Lambda}$ if $G$ is finitely presented relative to $\left\{H_{\lambda}\right\}_{\lambda \in \Lambda}$ and there is a constant $C>0$ such that for any word $W$ in $X \cup \mathcal{H}$ representing the identity in $G$, we have

$$
\operatorname{Area}^{r e l}(W) \leq C\|W\| .
$$

The constant $C$ in (5) is called an isoperimetric constant of the relative presentation (2) and $\left\{H_{\lambda}\right\}_{\lambda \in \Lambda}$ is called the collection of peripheral (or parabolic) subgroups of $G$. In particular, $G$ is an ordinary (Gromov) hyperbolic group if $G$ is hyperbolic relative to the trivial subgroup. Later on, by saying that a group $G$ is relatively hyperbolic, we will mean that there exists a collection of proper subgroups $\left\{H_{\lambda} \leq G \mid \lambda \in \Lambda\right\}$ such that $G$ is hyperbolic relative to $\left\{H_{\lambda}\right\}_{\lambda \in \Lambda}$.

This definition is independent of the choice of the finite generating set $X$ and the finite set $\mathcal{R}$ in (2) (see [33]).

Lemma 2.2 (33, Thm. 1.4). Let $G$ be a group hyperbolic relative to a collection of subgroups $\left\{H_{\lambda}\right\}_{\lambda \in \Lambda}$. Then the following conditions hold.

(1) For every $\lambda, \mu \in \Lambda, \lambda \neq \mu$, and every $g \in G$, we have $\left|H_{\lambda} \cap H_{\mu}^{g}\right|<\infty$.

(2) For every $\lambda \in \Lambda$ and $g \in G \backslash H_{\lambda}$, we have $\left|H_{\lambda} \cap H_{\lambda}^{g}\right|<\infty$.

Components. Let $G$ be a group hyperbolic relative to a family of proper subgroups $\left\{H_{\lambda}\right\}_{\lambda \in \Lambda}$. We recall some auxiliary terminology introduced in [33, which plays an important role in our paper.

Definition 2.3. Let $q$ be a path in the Cayley graph $\Gamma(G, X \cup \mathcal{H})$. A (non-trivial) subpath $p$ of $q$ is called an $H_{\lambda}$-component (or simply a component) if the label of $p$ is a word in the alphabet $H_{\lambda} \backslash\{1\}$ and $p$ is not contained in a longer subpath of $q$ with this property. Two $H_{\lambda}$-components $p_{1}, p_{2}$ of a path $q$ in $\Gamma(G, X \cup \mathcal{H})$ are called connected if there exists a path $c$ in $\Gamma(G, X \cup \mathcal{H})$ that connects some vertex of $p_{1}$ to some vertex of $p_{2}$, and $\operatorname{Lab}(c)$ is a word consisting of letters from $H_{\lambda} \backslash\{1\}$. In algebraic terms this means that all vertices of $p_{1}$ and $p_{2}$ belong to the same coset $g H_{\lambda}$ for a certain $g \in G$. Note that we can always assume that $c$ has length at most 1 , as every non-trivial element of $H_{\lambda} \backslash\{1\}$ is included in the set of generators.

Loxodromic elements and elementary subgroups. Recall that an element $g \in G$ is called parabolic if it is conjugate to an element of one of the subgroups $H_{\lambda}$, $\lambda \in \Lambda$. An element is said to be loxodromic if it is not parabolic and has infinite order. If $H$ is a subgroup of $G$, by $H^{0} \subset H$ we will denote the set of all elements of $H$ that are loxodromic in $G$.

Recall also that a group is elementary if it contains a cyclic subgroup of finite index. The next result was obtained in [31. The first part of the lemma is well known in the context of convergence groups 43. In particular, it follows from 43 . 
and 44] in case $G$ is finitely generated. (The latter assumption is only essential for 44.)

Lemma 2.4. Suppose a group $G$ is hyperbolic relative to a collection of subgroups $\left\{H_{\lambda}\right\}_{\lambda \in \Lambda}$. Let $g$ be a loxodromic element of $G$. Then the following conditions hold.

(a) There is a unique maximal elementary subgroup $E_{G}(g) \leq G$ containing $g$.

(b) $E_{G}(g)=\left\{h \in G \mid \exists m \in \mathbb{N}\right.$ such that $\left.h^{-1} g^{m} h=g^{ \pm m}\right\}$.

(c) The group $G$ is hyperbolic relative to the collection $\left\{H_{\lambda}\right\}_{\lambda \in \Lambda} \cup\left\{E_{G}(g)\right\}$.

For finitely generated relatively hyperbolic groups, a lemma similar to Lemma 2.4 (c) was also stated in 10. Namely it was claimed that if $G$ is a (finitely generated) relatively hyperbolic group and $Z$ is an infinite cyclic subgroup of $G$ such that $Z$ coincides with its normalizer, then $Z$ can be joined to the collection of peripheral subgroups of $G$ [10, Lemma 4.4]. We note that this is wrong even in case $G$ is an ordinary hyperbolic group.

The simplest counterexample is given by the group

$$
G=\left\langle z, c \mid c^{3}=1, z c z^{-1}=c^{2}\right\rangle
$$

and the subgroup $Z=\langle z\rangle$. Obviously $G$ splits as $1 \rightarrow C \rightarrow G \rightarrow \mathbb{Z} \rightarrow 1$, where $C=\langle c\rangle \cong \mathbb{Z} / 3 \mathbb{Z}$. In particular $G$ is hyperbolic (or, equivalently, hyperbolic relative to the trivial subgroup). It is straightforward to check that $Z$ coincides with its own normalizer in $G$. Indeed every element $g \in G$ has the form $z^{k} c^{m}$, where $k \in \mathbb{Z}$ and $m \in\{0,1,2\}$. If $m=1$, we have

$$
g^{-1} z g=\left(c^{-1} z^{-k}\right) z\left(z^{k} c\right)=c^{-1} z c=c^{-1}\left(z c z^{-1}\right) z=c^{-1} c^{2} z=c z \notin Z .
$$

Similarly $g^{-1} z g \notin Z$ if $m=2$. On the other hand, $G$ is not hyperbolic relative to $Z$. Indeed $c^{-1} z^{2} c=z^{2}$ and hence $Z \cap c^{-1} Z c$ is infinite. This contradicts part (b) of Lemma 2.2. Similarly for every (finitely generated) group $H$, the free product $G * H$ is hyperbolic relative to $H$, and the subgroup $Z$ provides a counterexample. Note that $E_{G}(z)=E_{G * H}(z)=G$, so applying Lemma 2.4 (c) yields the correct result.

Finite normal subgroups. The following result was proved in [3, Lemma 3.3].

Lemma 2.5. Let $H$ be a non-elementary subgroup of a relatively hyperbolic group G. Suppose that $H^{0} \neq \emptyset$. Then the subgroup $E_{G}(H)=\bigcap_{h \in H^{0}} E_{G}(h)$ is the (unique) maximal finite subgroup of $G$ normalized by $H$.

Corollary 2.6. Let $G$ be a relatively hyperbolic group. Then $G$ possesses a unique maximal finite normal subgroup $E(G)$.

Proof. If $G$ is finite, then the statement is trivial. If $G$ contains an infinite normal cyclic subgroup $C$ of finite index, then denote by $K$ the union of all finite normal subgroups of $G$. It is easy to see that $K$ is a torsion normal subgroup of $G$ (because a product of two finite normal subgroups is itself a finite normal subgroup). Since $K \cap C=\{1\}, K$ injects into the finite quotient $G / C$, hence $K$ is finite.

Finally, if $G$ is non-elementary, then $G^{0} \neq \emptyset$ by [31, Cor. 4.5] (if $G$ is finitely generated, this also follows from [43] and 44]). It remains to apply Lemma 2.5] to the case $G=H$. 


\section{SPECIAL ELEMENTS IN RELATIVELY HYPERBOLIC GROUPS}

Let $G$ be a relatively hyperbolic group and let $H$ be a non-elementary subgroup of $G$ containing at least one loxodromic element.

Definition 3.1. We say that an element $h \in H$ is $H$-special if $h$ is loxodromic in $G$ and $E_{G}(h)=\langle h\rangle \times E_{G}(H)$. The set of all $H$-special elements will be denoted by $S_{G}(H)$.

Note that, by definition, any $g \in S_{G}(H)$ belongs to the centralizer $C_{H}\left(E_{G}(H)\right)$. The result below was obtained in [3, Lemma 3.8].

Lemma 3.2. If $G$ is a relatively hyperbolic group and $H \leq G$ is a non-elementary subgroup such that $H^{0} \neq \emptyset$, then $S_{G}(H)$ is non-empty.

Special elements play a significant role in our approach to study automorphisms of relatively hyperbolic groups. They represent a useful tool that helps to deal with the technical problems which may arise when the group under consideration contains torsion. The main goal of this section is to prove the following important statement:

Proposition 3.3. Suppose that $G$ is a relatively hyperbolic group and $H \leq G$ is a non-elementary subgroup with $H^{0} \neq \emptyset$. Then $C_{H}\left(E_{G}(H)\right)$ is generated by the set $S_{G}(H)$. In particular, $\left\langle S_{G}(H)\right\rangle$ has finite index in $H$.

Observe that the statement after 'in particular' follows from the fact that the centralizer of a finite subgroup of $G$, normalized by $H$, necessarily has finite index in $H$.

We begin with some auxiliary results. Let $G$ be a group hyperbolic relative to a family of proper subgroups $\left\{H_{\lambda}\right\}_{\lambda \in \Lambda}$. If $G$ is infinite, it always contains a loxodromic element [31, Corollary 4.5]. The next lemma provides us with a tool for constructing such elements. It was proved in [31, Lemma 4.4].

Lemma 3.4. Let $G$ be a group hyperbolic relative to a collection of subgroups $\left\{H_{\lambda}\right\}_{\lambda \in \Lambda}$. For any $\lambda \in \Lambda$ and $a \in G \backslash H_{\lambda}$, there exists a finite subset $\mathcal{F} \subseteq H_{\lambda}$ such that if $h \in H_{\lambda} \backslash \mathcal{F}$, then ah is loxodromic.

Suppose that $\Xi$ is a finite subset of $G$. Define $\mathcal{W}(\Xi)$ to be the set of all words $W$ over the alphabet $X \cup \mathcal{H}$ that have the following form:

$$
W \equiv x_{0} h_{0} x_{1} h_{1} \ldots x_{l} h_{l} x_{l+1},
$$

where $l \in \mathbb{Z}, l \geq-2$ (if $l=-2$, then $W$ is the empty word; if $l=-1$, then $W \equiv x_{0}$ ), $h_{i}$ and $x_{i}$ are considered as single letters and

1) $x_{i} \in X \cup\{1\}, i=0, \ldots, l+1$, and for each $i=0, \ldots, l$, there exists $\lambda(i) \in \Lambda$ such that $h_{i} \in H_{\lambda(i)}$

2) if $\lambda(i)=\lambda(i+1)$, then $x_{i+1} \notin H_{\lambda(i)}$ for each $i=0, \ldots, l-1$;

3) $h_{i} \notin \Xi, i=0, \ldots, l$.

The statement below was proved in [27, Lemmas 6.3, 6.5].

Lemma 3.5. There is a finite subset $\Xi$ of $G$ such that the following holds. Suppose that $o=r q r^{\prime} q^{\prime}$ is a cycle in $\Gamma(G, X \cup \mathcal{H})$ with $\operatorname{Lab}(q), \operatorname{Lab}\left(q^{\prime}\right) \in \mathcal{W}(\Xi)$. Set $C=$ $\max \left\{\mathrm{L}(r), \mathrm{L}\left(r^{\prime}\right)\right\}$. 
(a) If $l$ is the number of components of $q$, then at least $(l-6 C)$ of components of $q$ are connected to components of $q^{\prime}$, and two distinct components of $q$ cannot be connected to the same component of $q^{\prime}$. A similar statement holds for $q^{\prime}$.

(b) For any $d \in \mathbb{N}$ there exists a constant $L=L(C, d) \in \mathbb{N}$ such that if $\mathrm{L}(q) \geq L$, then there are $d$ consecutive components $p_{s}, \ldots, p_{s+d-1}$ of $q$ and $p_{s^{\prime}}^{\prime}, \ldots, p_{s^{\prime}+d-1}^{\prime}$ of $q^{\prime-1}$, so that $p_{s+i}$ is connected to $p_{s^{\prime}+i}^{\prime}$ for each $i=0, \ldots, d-1$.

Proposition 3.3 is an easy consequence of Lemma 3.6 below. In the case when $G$ is an ordinary word hyperbolic group it was proved in [28, Lemma 4.3].

Lemma 3.6. Suppose that $g \in S_{G}(H)$ and $x \in C_{H}\left(E_{G}(H)\right) \backslash E_{G}(g)$. Then there exists $N_{1} \in \mathbb{N}$ such that $g^{n} x \in S_{G}(H)$ for any $n \in \mathbb{Z}$ with $|n| \geq N_{1}$.

Proof. By part (3) of Lemma 2.4 $G$ is hyperbolic relative to the collection of subgroups $\left\{H_{\lambda}\right\}_{\lambda \in \Lambda} \cup\left\{E_{G}(g)\right\}$. Denote $\mathcal{H}^{\prime}=\left(\bigcup_{\lambda \in \Lambda} H_{\lambda} \cup E_{G}(g)\right) \backslash\{1\} \subset G$. After adding $x$ and $x^{-1}$ to the finite relative generating set of $G$, if necessary, we can assume that $x^{ \pm 1} \in X$. Let $\mathcal{F}$ and $\Xi$ be the finite subsets of $G$ given by Lemmas 3.4 and 3.5 respectively. Since $g$ has infinite order, there exists $N_{1} \in \mathbb{N}$ such that $g^{n} \notin \mathcal{F} \cup \Xi$ for any $n \in \mathbb{Z}$ with $|n| \geq N_{1}$.

Choose an arbitrary $n \in \mathbb{Z}$ such that $|n| \geq N_{1}$. By Lemma 3.4, the element $g^{n} x=\left(x g^{n}\right)^{x}$ is loxodromic in $G$. Suppose that $y \in E_{G}\left(g^{n} x\right)$. By part (2) of Lemma 2.4, there are $m \in \mathbb{N}$ and $\epsilon \in\{-1,1\}$ such that

$$
y\left(g^{n} x\right)^{m} y^{-1}=\left(g^{n} x\right)^{\epsilon m} .
$$

Let $V$ be the letter from $\mathcal{H}^{\prime}$ representing $g^{n}$ in $G$, let $W$ be the letter from $X$ representing $x$, and let $U$ be the shortest word over the alphabet $X \cup \mathcal{H}^{\prime}$ representing $y$. Set $C=\|U\|$ and $d=1$. Now we apply Lemma 3.5(b) to find the constant $L=L(C, d)$ from its claim. Evidently we can assume that the number $m$ from equation (6) is larger than $L$.

Consider a cycle $o=r q r^{\prime} q^{\prime}$ in $\Gamma\left(G, X \cup \mathcal{H}^{\prime}\right)$, where $\operatorname{Lab}(r) \equiv U, \operatorname{Lab}(q) \equiv$ $(V W)^{m}, \operatorname{Lab}\left(r^{\prime}\right) \equiv U^{-1}, \operatorname{Lab}\left(q^{\prime}\right) \equiv(V W)^{-\epsilon m}$. By construction, the cycle $o$ satisfies the assumptions of Lemma 3.5(b), hence some components $p$ of $q$ and $p^{\prime}$ of $q^{\prime-1}$ must be connected in $\Gamma\left(G, X \cup \mathcal{H}^{\prime}\right)$. That is, there is a path $s$ with $s_{-}=p_{+}, s_{+}=p_{+}^{\prime}$ and $z=\operatorname{Lab}(s) \in E_{G}(g)$ (see Figure 1). Note that $\operatorname{Lab}(p) \equiv V, \operatorname{Lab}\left(p^{\prime}\right) \equiv V^{\epsilon}$.

Let $q_{1}$ be the subpath of $q$ starting at $r_{+}=q_{-}$and ending at $p_{+}=s_{-}$; let $q_{1}^{\prime}$ be the subpath of $q^{\prime}$ starting at $s_{+}=p_{+}^{\prime}$ and ending at $q_{+}^{\prime}=r_{-}$. Considering the cycle $o_{1}=r q_{1} s q_{1}^{\prime}$ in the case when $\epsilon=-1$ we get the following equality in $G$ :

$$
\left(g^{n} x\right)^{\xi} y\left(g^{n} x\right)^{\zeta}=z^{-1} g^{-n} \in E_{G}\left(g^{n} x\right) \cap E_{G}(g) \text { for some } \xi, \zeta \in \mathbb{Z} .
$$

Similarly, in the case when $\epsilon=1$, we get

$$
\left(g^{n} x\right)^{\xi} y\left(g^{n} x\right)^{\zeta}=g^{n} z^{-1} g^{-n} \in E_{G}\left(g^{n} x\right) \cap E_{G}(g) \text { for some } \xi, \zeta \in \mathbb{Z} .
$$

Observe that by Lemma 2.4 the group $G$ is hyperbolic relative to $\left\{H_{\lambda}\right\}_{\lambda \in \Lambda} \cup$ $\left\{E_{G}(g), E_{G}\left(g^{n} x\right)\right\}$, hence, by Lemma 2.2, the intersection $E_{G}\left(g^{n} x\right) \cap E_{G}(g)$ is finite. Since $g$ is $H$-special, any finite subgroup of $E_{G}(g)$ is contained in $E_{G}(H)$. Therefore $E_{G}\left(g^{n} x\right) \cap E_{G}(g) \subset E_{G}(H)$. Thus, whatever $\epsilon \in\{-1,1\}$ is, we always have $\left(g^{n} x\right)^{\xi} y\left(g^{n} x\right)^{\zeta}=h \in E_{G}(H)$, implying that $y=\left(g^{n} x\right)^{-\xi-\zeta} h$ because $g, x \in C_{H}\left(E_{G}(H)\right)$. By part (2) of Lemma 2.4, $\left\langle g^{n} x\right\rangle$ and $E_{G}(H)$ are both contained in $E_{G}\left(g^{n} x\right)$; consequently $E_{G}\left(g^{n} x\right)=\left\langle g^{n} x\right\rangle \times E_{G}(H)$. 


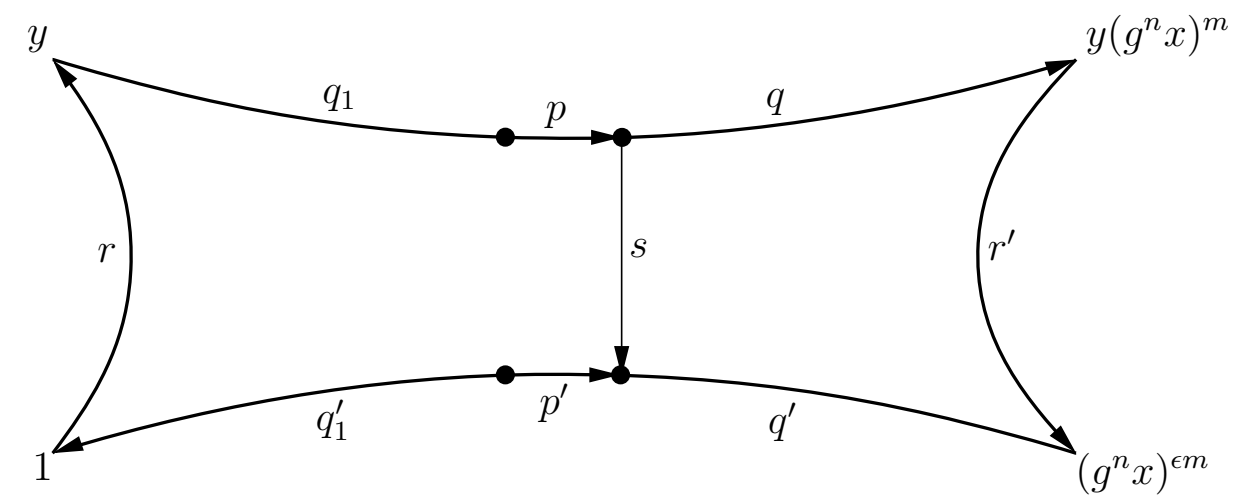

FigURE 1

Proof of Proposition 3.3. By Lemma 3.2 we can find an element $g \in S_{G}(H)$. Note that for any $x \in Z=E_{G}(H) \cap C_{H}\left(E_{G}(H)\right)$, the element $g x$ is also $H$-special. Since $x=g^{-1}(g x)$, we have $Z \subset\left\langle S_{G}(H)\right\rangle$. It is easy to see that $E_{G}(g) \cap C_{H}\left(E_{G}(H)\right)=$ $\langle g\rangle \times Z$, hence $E_{G}(g) \cap C_{H}\left(E_{G}(H)\right) \subset\left\langle S_{G}(H)\right\rangle$. Now, if $x \in C_{H}\left(E_{G}(H)\right) \backslash E_{G}(g)$, then by Lemma 3.6, $g^{n} x \in S_{G}(H)$ for some $n \in \mathbb{N}$. Consequently, $x=g^{-n}\left(g^{n} x\right) \in$ $\left\langle S_{G}(H)\right\rangle$.

\section{Technical Lemmas}

Our main goal here is to prove several auxiliary lemmas, which will be used in the next section to give an algebraic description of automorphisms preserving commensurability classes of elements in relatively hyperbolic groups. We begin with a definition.

Definition 4.1. Let $G$ be a group. Two elements $x, y \in G$, are said to be commensurable if there are $z \in G, m, n \in \mathbb{Z} \backslash\{0\}$ such that $y^{n}=z x^{m} z^{-1}$ in $G$. If the elements $x$ and $y$ are commensurable in $G$, we will write $x \stackrel{G}{\approx} y$; otherwise, we will write $x \stackrel{G}{\not} y$.

Remark 4.2. Obviously any two elements of finite order are commensurable. Further, if $g$ and $h$ are commensurable elements of a relatively hyperbolic group $G$ and $g$ is loxodromic, then $h$ is loxodromic too. Indeed, evidently $h$ has infinite order. Suppose that $h$ is parabolic. Since $g \stackrel{G}{\approx} h$, there are $\lambda \in \Lambda, a \in G$ and $m \in \mathbb{N}$ such that $a^{-1} g^{m} a \in H_{\lambda}$. Since $g$ is loxodromic, $x=g^{a} \notin H_{\lambda}$ and the intersection $H^{x} \cap H$ contains an infinite order element $x^{m}$. The latter contradicts claim (2) of Lemma 2.2

Throughout the rest of this section, $G$ will denote a group hyperbolic relative to a collection of peripheral subgroups $\left\{H_{\lambda}\right\}_{\lambda \in \Lambda}$, and $H \leq G$ will denote a nonelementary subgroup with $H^{0} \neq \emptyset$.

Lemma 4.3. Let $g \in G$ be a loxodromic element and $x \in G \backslash E_{G}(g)$. For any finite subset $Y$ of $G$ there is $N_{2} \in \mathbb{N}$ such that $g^{n} x$ is loxodromic and is not commensurable with any $y \in Y$ whenever $|n| \geq N_{2}$. 
Proof. In view of Lemma 2.4(3), we can assume that $E_{G}(g)$ belongs to the family of peripheral subgroups $\left\{H_{\lambda}\right\}_{\lambda \in \Lambda}$ of $G$ and each infinite order element $y \in Y$ is parabolic.

Now we can apply Lemma 3.4 to find $N_{2} \in \mathbb{N}$ such that for any $n \in \mathbb{Z}$ with $|n| \geq N_{2}$, the element $x g^{n}$ is loxodromic. Therefore, so is $h=g^{n} x=x^{-1}\left(x g^{n}\right) x$. Suppose that $h$ is commensurable with some $y \in Y$. Then $y$ must also be loxodromic (by Remark 4.2), which contradicts our assumption above.

Lemma 4.4. Let $\left\{g_{1}, \ldots, g_{l}\right\}, l \geq 2$, be a finite set of pairwise non-commensurable loxodromic elements in a relatively hyperbolic group $G$. For any finite subset $F \subset G$ there exists $N_{3} \in \mathbb{N}$ such that for any permutation $\sigma$ of $\{1,2, \ldots, l\}$ and arbitrary elements $h_{i} \in E_{G}\left(g_{\sigma(i)}\right), i=1,2, \ldots, l$, of infinite order, the following hold.

(i) The element $g=g_{1}^{m_{1}} f_{1} g_{2}^{m_{2}} f_{2} \ldots g_{l}^{m_{l}} f_{l}$ is loxodromic for any $f_{i} \in F$ and $m_{i} \in \mathbb{Z}$ with $\left|m_{i}\right| \geq N_{3}, i=1,2, \ldots, l$.

(ii) Suppose that $\left(g_{1}^{m_{1}} g_{2}^{m_{2}} \ldots g_{l}^{m_{l}}\right)^{\zeta}$ is conjugate to $\left(h_{1}^{n_{1}} f_{1} h_{2}^{n_{2}} f_{2} \ldots h_{l}^{n_{l}} f_{l}\right)^{\eta}$ in $G$, for some $f_{i} \in F, \zeta, \eta \in \mathbb{N}, m_{i}, n_{i} \in \mathbb{Z},\left|m_{i}\right| \geq N_{3},\left|n_{i}\right| \geq N_{3}$ for all $i=$ $1,2, \ldots, l$. Then $\zeta=\eta$, there is $k \in\{0,1, \ldots, l-1\}$ such that $\sigma$ is a cyclic shift by $k$, that is, $\sigma(i) \equiv i+k(\bmod l)$ for all $i \in\{1,2, \ldots, l\}$, and $f_{j} \in$ $E_{G}\left(g_{\sigma(j)}\right) E_{G}\left(g_{\sigma(j+1)}\right)$ when $j=1,2, \ldots, l-1, f_{l} \in E_{G}\left(g_{\sigma(l)}\right) E_{G}\left(g_{\sigma(1)}\right)$.

Proof. By Lemma 2.4 and because $g_{i} \not ̈ g_{j}$ when $i \neq j, G$ is hyperbolic relative to the extended collection of subgroups $\left\{H_{\lambda}\right\}_{\lambda \in \Lambda} \cup\left\{E_{G}\left(g_{i}\right)\right\}_{i=1}^{l}$. Also, the finite relative generating set $X$ can be replaced by the bigger finite set $X^{\prime}=X \cup F \cup F^{-1}$ retaining the relative hyperbolicity of $G$. Denote $\mathcal{H}^{\prime}=\left(\bigcup_{\lambda \in \Lambda} H_{\lambda} \cup \bigcup_{i=1}^{l} E_{G}\left(g_{i}\right)\right) \backslash$ $\{1\} \subset G$. Let $\Xi$ be the finite subset of $G$ given by Lemma 3.5.

Take any $i \in\{1, \ldots, l\}$. By part (1) of Lemma 2.4, we have $\left|E_{G}\left(g_{i}\right):\left\langle g_{i}\right\rangle\right|<\infty$, hence any infinite order element $h \in E_{G}\left(g_{i}\right)$ belongs to the elementary subgroup

$$
E_{G}^{+}\left(g_{i}\right)=\left\{x \in G \mid \exists m \in \mathbb{N} \text { such that } x^{-1} g_{i}^{m} x=g_{i}^{m}\right\} \leq E_{G}\left(g_{i}\right) .
$$

Clearly, the center of $E_{G}^{+}\left(G_{i}\right)$ has finite index in it, hence all finite order elements of $E_{G}^{+}\left(g_{i}\right)$ form the maximal torsion subgroup $T \triangleleft E_{G}^{+}\left(g_{i}\right)$. Let $\alpha: E_{G}^{+}\left(g_{i}\right) \rightarrow E_{G}^{+}\left(g_{i}\right) / T$ be the natural epimorphism. The image $\alpha\left(E_{G}^{+}\left(g_{i}\right)\right)$ is infinite cyclic (because it is virtually cyclic and torsion-free), therefore there exists $K_{i} \in \mathbb{N}$ such that for every non-trivial element $y \in \alpha\left(E_{G}^{+}\left(g_{i}\right)\right)$, one has $y^{n} \notin S_{i}$ whenever $|n| \geq K_{i}$, where $S_{i}=\alpha\left(E_{G}^{+}\left(g_{i}\right) \cap \Xi\right)$ is a finite subset of $\alpha\left(E_{G}^{+}\left(g_{i}\right)\right)$. Set $N_{3}=\max \left\{K_{i} \mid i=1, \ldots, l\right\}$. By construction, for every $i$ and each infinite order element $h \in E_{G}\left(g_{i}\right)$, we have $h^{n} \notin \Xi$ whenever $|n| \geq N_{3}$.

Choose any elements $f_{i} \in F$ and integers $m_{i}$ with $\left|m_{i}\right| \geq N_{3}, i=1, \ldots, l$. Let $V_{i}$ and $W_{i}$ be the letters from $\mathcal{H}^{\prime}$ and from $X^{\prime}$ representing the elements $g_{i}^{m_{i}}$ and $f_{i}, i=1, \ldots, l$, respectively.

Proving claim (i) by contradiction, suppose that the element $g$ is not loxodromic.

If $g$ has finite order $t \in \mathbb{N}$, then set $C=0, d=1$ and choose $L=L(C, d)$ according to Lemma 3.5(b). In the Cayley graph $\Gamma\left(G, X^{\prime} \cup \mathcal{H}^{\prime}\right)$ consider the cycle $o=r q r^{\prime} q^{\prime}$, where $\operatorname{Lab}(q) \equiv\left(V_{1} W_{1} V_{2} W_{2} \ldots V_{l} W_{l}\right)^{L t}$, and $r, r^{\prime}$ and $q^{\prime}$ are trivial paths consisting of a single vertex $q_{-}=q_{+}=1$. Since $\mathrm{L}(q) \geq L t \geq L$, it follows from Lemma 3.5 (b) that some component of $q$ must be connected to a component of $q^{\prime-1}$. But $q^{\prime-1}$ has no components at all, a contradiction. 
Therefore $g$ must have infinite order and must be parabolic, i.e., $g=a h a^{-1}$ for some $h \in \mathcal{H}^{\prime}$ and $a \in G$. Let $C=|a|_{X^{\prime} \cup \mathcal{H}^{\prime}}, d=2$ and $L=L(C, d)$ be given by Lemma 3.5(b). Since $h$ has infinite order (as a conjugate of $g$ ), there is $n \in \mathbb{N}$ such that $n \geq L$ and $h^{n} \notin \Xi$. Choose a shortest word $A$ over $X^{\prime} \cup \mathcal{H}^{\prime}$ representing $a$ in $G$, and let $U$ be the letter from $\mathcal{H}^{\prime}$ corresponding to $h^{n}$. Consider a cycle $o=r q r^{\prime} q^{\prime}$ in $\Gamma\left(G, X^{\prime} \cup \mathcal{H}^{\prime}\right)$ such that $\operatorname{Lab}(r) \equiv A, q_{-}=r_{+}, \operatorname{Lab}(q) \equiv\left(V_{1} W_{1} V_{2} W_{2} \ldots V_{l} W_{l}\right)^{n}$, $r_{-}^{\prime}=q_{+}, \operatorname{Lab}\left(r^{\prime}\right) \equiv A^{-1}, q_{-}^{\prime}=r_{+}^{\prime}, \operatorname{Lab}\left(q^{\prime}\right) \equiv U^{-1}$. Since $\mathrm{L}(r)=\mathrm{L}\left(r^{\prime}\right)=C, \mathrm{~L}(q) \geq$ $n \geq L$, we can apply Lemma 3.5. (b) to $o$, claiming that two distinct components of $q$ must be connected to two distinct components of $q^{\prime-1}$. But $q^{\prime-1}$ has only one component by definition. This contradiction concludes the proof of claim (i).

To establish claim (ii), assume that $b\left(g_{1}^{m_{1}} g_{2}^{m_{2}} \ldots g_{l}^{m_{l}}\right)^{\zeta} b^{-1}=\left(h_{1}^{n_{1}} f_{1} h_{2}^{n_{2}} f_{2} \ldots\right.$ $\left.h_{l}^{n_{l}} f_{l}\right)^{\eta}$ in $G$, for some infinite order elements $h_{i} \in E_{G}\left(g_{\sigma(i)}\right), b \in G, \zeta, \eta \in \mathbb{N}$, $m_{i}, n_{i} \in \mathbb{Z},\left|m_{i}\right| \geq N_{3},\left|n_{i}\right| \geq N_{3}$ for $i=1,2, \ldots, l$. Then for every $\varkappa \in \mathbb{N}$ we have

$$
b\left(g_{1}^{m_{1}} g_{2}^{m_{2}} \ldots g_{l}^{m_{l}}\right)^{\varkappa \zeta} b^{-1}=\left(h_{1}^{n_{1}} f_{1} h_{2}^{n_{2}} f_{2} \ldots h_{l}^{n_{l}} f_{l}\right)^{\varkappa \eta} .
$$

Let $V_{i}$ and $W_{i}$ be as before. Choose a letter $U_{i}$ from $\mathcal{H}^{\prime}$ corresponding to $h_{i}^{n_{i}}$, $i=1, \ldots, l$, and a shortest word $B$ over $X^{\prime} \cup \mathcal{H}^{\prime}$ representing $b$ in $G$. Set $C=\|B\|$, $d=2 l$ and let $L=L(C, d) \in \mathbb{N}$ be the constant given by Lemma [3.5)(b). Take $\varkappa \in \mathbb{N}$ so that $\varkappa \zeta l \geq L$ and $\varkappa l>6 C$.

In the Cayley graph $\Gamma\left(G, X^{\prime} \cup \mathcal{H}^{\prime}\right)$ equation (17) gives rise to a cycle $o=r q r^{\prime} q^{\prime}$, in which $\operatorname{Lab}(r) \equiv B, q_{-}=r_{+}, \operatorname{Lab}(q) \equiv\left(V_{1} V_{2} \ldots V_{l}\right)^{\varkappa \zeta}, r_{-}^{\prime}=q_{+}, \operatorname{Lab}\left(r^{\prime}\right) \equiv B^{-1}$, $q_{-}^{\prime}=r_{+}^{\prime}, \operatorname{Lab}\left(q^{\prime}\right) \equiv\left(U_{1} W_{1} U_{2} W_{2} \ldots U_{l} W_{l}\right)^{-\varkappa \eta}$.

By construction, the paths $q$ and $q^{\prime}$ have exactly $\varkappa \zeta l$ and $\varkappa \eta l$ components, respectively. Suppose that $\zeta>\eta$. By Lemma 3.5) (a), at least $\varkappa \zeta l-6 C>\varkappa l(\zeta-$ $1) \geq \varkappa l \eta$ components of $q$ must be connected to components of $q^{\prime}$, hence two distinct components of $q$ will have to be connected to the same component of $q^{\prime}$, contradicting Lemma [3.5(a). Hence $\zeta \leq \eta$. A symmetric argument shows that $\eta \leq \zeta$. Consequently $\zeta=\eta$.

Since $\mathrm{L}(q)=\varkappa \zeta l \geq \mathrm{L}$, we can apply Lemma 3.5(b) to find $2 l$ consecutive components of $q$ that are connected to $2 l$ consecutive components of $q^{\prime-1}$. Therefore there are consecutive components $p_{1}, \ldots, p_{l+1}$ of $q$ and $p_{1}^{\prime}, \ldots, p_{l+1}^{\prime}$ of $q^{\prime-1}$ such that $p_{j}$ is connected to $p_{j}^{\prime}$ for each $j$, and $\operatorname{Lab}\left(p_{i}\right) \equiv V_{i}$ for $i=1, \ldots, l, \operatorname{Lab}\left(p_{l+1}\right) \equiv V_{1}$ (Figure 2). Therefore $\operatorname{Lab}\left(p_{i}^{\prime}\right) \in E_{G}\left(g_{i}\right), i=1, \ldots, l, \operatorname{Lab}\left(p_{l+1}^{\prime}\right) \in E_{G}\left(g_{1}\right)$. From the form of $\operatorname{Lab}\left(q^{\prime-1}\right)$ it follows that there is $k \in\{0,1, \ldots, l-1\}$ such that $\operatorname{Lab}\left(p_{j}^{\prime}\right) \equiv U_{j+k}$ for $j=1, \ldots, l+1$ (indices at $U$ are taken modulo $l$ ). Thus $U_{j+k}=h_{j+k}^{n_{j+k}} \in E_{G}\left(g_{j}\right)$. On the other hand, $h_{j+k}^{n_{j+k}} \in E_{G}\left(g_{\sigma(j+k)}\right)$ has infinite order. Hence the intersection $E_{G}\left(g_{j}\right) \cap E_{G}\left(g_{\sigma(j+k)}\right)$ must be infinite, which yields (by Lemma 2.2) that $\sigma(j+k)=j$ for all $j$. Therefore $\sigma$ is a cyclic shift (by $l-k$ ) of $\{1, \ldots, l\}$.

The subpath $w_{i}$ of $q^{\prime-1}$ between $\left(p_{i}^{\prime}\right)_{+}$and $\left(p_{i+1}^{\prime}\right)_{-}$is labelled by $W_{\sigma^{-1}(i)}$. As we showed, the vertex $\left(p_{i}\right)_{+}=\left(p_{i+1}\right)_{-}$is connected to $\left(w_{i}\right)_{-}$by a path $s_{i}$ with $\operatorname{Lab}\left(s_{i}\right) \in E_{G}\left(g_{i}\right)$, and to $\left(w_{i}\right)_{+}$by a path $t_{i}$ with $\operatorname{Lab}\left(t_{i}\right) \in E_{G}\left(g_{i+1}\right), i=1, \ldots, l$ (here we use the convention that $g_{l+1}=g_{1}$ ). Considering the cycle $t_{i}^{-1} s_{i} w_{i}$ we achieve the desired inclusion: $f_{\sigma^{-1}(i)}=\operatorname{Lab}\left(w_{i}\right) \in E_{G}\left(g_{i}\right) E_{G}\left(g_{i+1}\right), i=1, \ldots, l$. This concludes the proof.

Lemma 4.5. Suppose that $\varphi: H \rightarrow G$ is a homomorphism such that $\varphi(h) \stackrel{G}{\approx} h$ for all $h \in H^{0}$. Then for any $g_{1}, g_{2}, g_{3} \in H^{0}$, satisfying $g_{i} \underset{\sim}{\not} g_{j}$ for $i \neq j$, there exists 


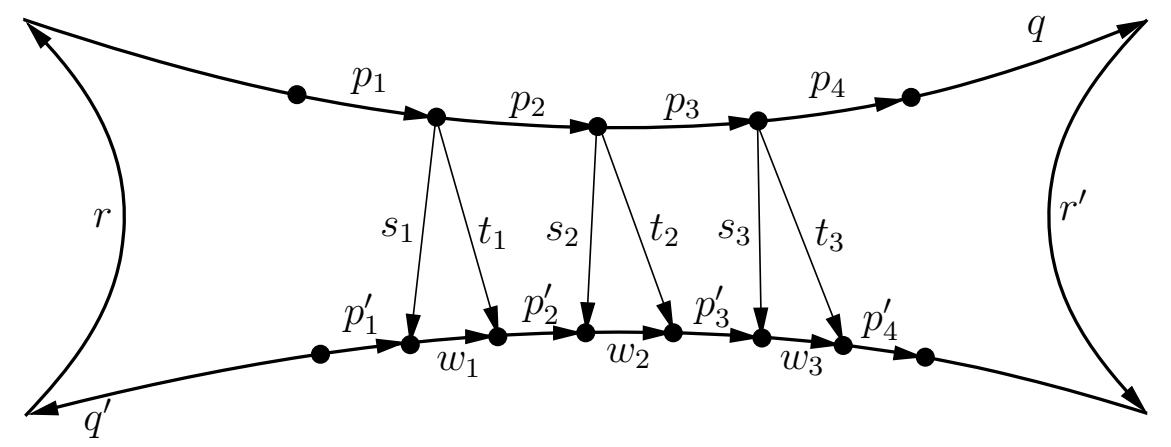

Figure 2

$N_{4} \in \mathbb{N}$ such that for arbitrary $n_{1}, n_{2}, n_{3} \in \mathbb{Z}$, with $\left|n_{i}\right| \geq N_{4}, i=1,2,3$, and for $g=g_{1}^{n_{1}} g_{2}^{n_{2}} g_{3}^{n_{3}}$, one has $g \in H^{0}$ and $(\varphi(g))^{\zeta}=e g^{\zeta} e^{-1}$, for some $e \in G$ and $\zeta \in \mathbb{N}$.

Proof. According to the assumptions, there exist $x_{i} \in G$ and $\zeta_{i}, \eta_{i} \in \mathbb{Z} \backslash\{0\}$ such that $\left(\varphi\left(g_{i}\right)\right)^{\zeta_{i}}=x_{i} g_{i}^{\eta_{i}} x_{i}^{-1}, i=1,2,3$. Denote $h_{i}=x_{i}^{-1} \varphi\left(g_{i}\right) x_{i}, i=1,2,3$. Then $h^{\zeta_{i}}=g^{\eta_{i}}$, hence $h_{i} \in E_{G}\left(g_{i}\right)$ (by part (2) of Lemma 2.4) and $h_{i}$ has infinite order, $i=1,2,3$.

Set $f_{1}=x_{1}^{-1} x_{2}, f_{2}=x_{2}^{-1} x_{3}$ and $f_{3}=x_{3}^{-1} x_{1}$, and let $N_{4} \in \mathbb{N}$ be the number $N_{3}$ from the claim of Lemma 4.4 applied to the set of loxodromic elements $\left\{g_{1}, g_{2}, g_{3}\right\}$ and the finite set $F=\left\{f_{1}, f_{2}, f_{3}\right\}$. Take any $n_{i} \in \mathbb{Z}$ with $\left|n_{i}\right| \geq N_{4}, i=1,2,3$. By part (i) of Lemma 4.4 $g=g_{1}^{n_{1}} g_{2}^{n_{2}} g_{3}^{n_{3}} \in H^{0}$. Hence there are $\zeta, \eta \in \mathbb{Z} \backslash\{0\}$ and $e \in G$ such that $e g^{\zeta} e^{-1}=(\varphi(g))^{\eta}$. Since $\varphi$ is a homomorphism, we get

$$
e\left(g_{1}^{n_{1}} g_{2}^{n_{2}} g_{3}^{n_{3}}\right)^{\zeta} e^{-1}=(\varphi(g))^{\eta}=\left(x_{1} h_{1}^{n_{1}} x_{1}^{-1} x_{2} h_{2}^{n_{2}} x_{2}^{-1} x_{3} h_{3}^{n_{3}} x_{3}^{-1}\right)^{\eta} \text {, hence }
$$

$$
\left(x_{1}^{-1} e\right)\left(g_{1}^{n_{1}} g_{2}^{n_{2}} g_{3}^{n_{3}}\right)^{\zeta}\left(x_{1}^{-1} e\right)^{-1}=\left(h_{1}^{n_{1}} f_{1} h_{2}^{n_{2}} f_{2} h_{3}^{n_{3}} f_{3}\right)^{\eta} .
$$

Without loss of generality we can assume that $\zeta>0$. Suppose that $\eta<0$. Then $\left(g_{3}^{-n_{3}} g_{2}^{-n_{2}} g_{1}^{-n_{1}}\right)^{\zeta}$ is conjugate to $\left(h_{1}^{n_{1}} f_{1} h_{2}^{n_{2}} f_{2} h_{3}^{n_{3}} f_{3}\right)^{-\eta}$ in $G$ and $-\eta>0$. Applying part (ii) of Lemma 4.4 to this situation, we get a contradiction with the fact that the transposition $(1,3)$ is not a cyclic shift of $\{1,2,3\}$. Therefore, $\eta>0$ and we can apply part (ii) of Lemma 4.4 to (8), achieving the required equality $\zeta=\eta$.

Lemma 4.6. Let $a, b \in G$ be non-commensurable loxodromic elements and let $y, z \in$ $G$. There exists $N_{5} \in \mathbb{N}$ such that the following holds. Suppose that $a^{k^{\prime}} y b^{l^{\prime}} z \stackrel{G}{\approx} a^{k} b^{l}$ for some integers $k, l, k^{\prime}, l^{\prime}$ with $|k|,|l|,\left|k^{\prime}\right|,\left|l^{\prime}\right| \geq N_{5}$. Then $y \in E_{G}(a) E_{G}(b)$ and $z \in E_{G}(b) E_{G}(a)$.

Proof. Choose $N_{5} \in \mathbb{N}$ to be the number $N_{3}$ arising after an application of Lemma 4.4 to $\{a, b\}$ and $F=\{y, z\}$. Choose any $k, l, k^{\prime}, l^{\prime} \in \mathbb{Z}$ satisfying $|k|,|l|,\left|k^{\prime}\right|,\left|l^{\prime}\right| \geq$ $N_{5}$.

Assume that there is $e \in G, \zeta \in \mathbb{N}$ and $\eta \in \mathbb{Z} \backslash\{0\}$ for which $e\left(a^{k} b^{l}\right)^{\zeta} e^{-1}=$ $\left(a^{k^{\prime}} y b^{l^{\prime}} z\right)^{\eta}$. If $\eta>0$, then the statement immediately follows from part (ii) of Lemma 4.4. So, suppose that $\eta<0$. Then $-\eta>0$ and $\left(b^{-l} a^{-k}\right)^{\zeta}$ is conjugate 
to $\left(a^{k^{\prime}} y b^{l^{\prime}} z\right)^{-\eta}$ in $G$. Again, by part (ii) of Lemma 4.4 $y \in E_{G}(a) E_{G}(b), z \in$ $E_{G}(b) E_{G}(a)$.

Lemma 4.7. Assume that $g \in S_{G}(H)$ and $\psi: H \rightarrow G$ is a homomorphism satisfying $\psi\left(g^{n}\right)=g^{n} z$ for some $n \in \mathbb{N}$ and $z \in E_{G}(H)$. Then there is $f \in E_{G}(H)$ such that $\psi(g)=g f$.

Proof. After replacing $n$ with $n^{\prime}=n\left|E_{G}(H)\right|$, we can further assume that $z=1$, because $\psi\left(g^{n^{\prime}}\right)=g^{n^{\prime}} z^{n^{\prime}}=g^{n^{\prime}}$.

Now, note that $\psi(g) g^{n}(\psi(g))^{-1}=\psi\left(g^{n}\right)=g^{n}$, hence $\psi(g) \in E_{G}(g)$ by part (2) of Lemma 2.4. Since $g$ is $H$-special, there is $k \in \mathbb{Z}$ and $f \in E_{G}(H)$ such that $\psi(g)=g^{k} f$. Denote $l=\left|E_{G}(H)\right|$. Then $g^{l n}=\psi\left(g^{l n}\right)=\left(g^{k} f\right)^{l n}=g^{l n k} f^{l n}=g^{l n k}$, implying that $k=1$, as required.

Lemma 4.8. Suppose that for an automorphism $\alpha \in$ Aut $(H)$ there is $g \in H^{0}$ satisfying $g \stackrel{G}{\not} \alpha(g)$. Then there exists an element $a \in H$ such that both a and $\alpha(a)$ are loxodromic in $G$ and $a \not{z} \alpha(a)$.

Proof. If $\alpha(g) \in H^{0}$, there is nothing to prove. Thus, we can assume that $\alpha(g)$ is parabolic in $G$; i.e., there exists a peripheral subgroup $H_{\lambda}$ and elements $t \in$ $G, h \in H_{\lambda}$ such that $\alpha(g)=h^{t}$. Denote $x=\alpha^{-1}(g) \in H$. If $x \in E_{G}(g)$, then $\langle g\rangle^{x} \cap\langle g\rangle$ is infinite (by Lemma 2.4(b)) hence $\langle\alpha(g)\rangle^{\alpha(x)} \cap\langle\alpha(g)\rangle$ is infinite. Thus $H_{\lambda}^{\left(t g t^{-1}\right)} \cap H_{\lambda}$ is infinite, which implies, by Lemma 2.2 that $\operatorname{tgt}^{-1} \in H_{\lambda}$, contradicting the loxodromicity of $g$.

Therefore $x \notin E_{G}(g)$. Since both $g$ and $\alpha(g)$ have infinite order and $y=t g t^{-1} \in$ $G \backslash H_{\lambda}$, we can apply Lemmas 4.3 and 3.4 to find $N \in \mathbb{N}$ such that for any integer $n \geq N$, the elements $g^{n} x$ and $h^{n} y$ are loxodromic in $G$. Note that $\alpha\left(g^{n} x\right)=\left(h^{n} y\right)^{t}$.

Suppose, first, that

$$
g^{n} x \stackrel{G}{\approx} \alpha\left(g^{n} x\right) \text { for every } n \geq N .
$$

By Lemma 2.4, $G$ is hyperbolic relative to $\left\{H_{\lambda}\right\}_{\lambda \in \Lambda} \cup\left\{E_{G}(g)\right\}$. Without loss of generality, we can also assume that $x$ and $y$ belong to the finite relative generating set $X$ of $G$. Let $\Xi \subset G$ be the finite set from Lemma 3.5. Evidently there is an integer $n \geq N$ such that $g^{n}, h^{n} \notin \Xi$. Our assumption (9) implies that there is $b \in G, k, l \in \mathbb{Z} \backslash\{0\}$ such that $b\left(g^{n} x\right)^{k} b^{-1}=\left(h^{n} y\right)^{l}$. Choose a word $B$ in the alphabet $X \cup \mathcal{H}^{\prime}$ representing $b$ in $G$, where $\mathcal{H}^{\prime}=\left(\bigcup_{\lambda \in \Lambda} H_{\lambda} \cup E_{G}(g)\right) \backslash\{1\}$, and let $W, Y \in X, U \in E_{G}(g), V \in H_{\lambda}$ be the letters corresponding to $x, y, g^{n}, h^{n}$, respectively. Set $d=1, C=\|B\|$ and let $L=L(C, d)$ be the constant provided by part (b) of Lemma 3.5. Without loss of generality we can assume that $|k|,|l| \geq L$.

Consider a cycle $o=r q r^{\prime} q^{\prime}$ in the Cayley graph $\Gamma\left(G, X \cup \mathcal{H}^{\prime}\right)$, where $\operatorname{Lab}(r) \equiv B$, $r_{+}=q_{-}, \operatorname{Lab}(q) \equiv(U W)^{k}, q_{+}=r_{-}^{\prime}, \operatorname{Lab}\left(r^{\prime}\right) \equiv B^{-1}, q_{-}^{\prime}=r_{+}^{\prime}$ and $\operatorname{Lab}\left(q^{\prime}\right) \equiv$ $(V Y)^{-l}$. It is easy to see that $o$ satisfies all the conditions of Lemma 3.5. hence some component of $q$ must be connected to a component of $q^{\prime-1}$ in $\Gamma\left(G, X \cup \mathcal{H}^{\prime}\right)$. However, according to the construction, $q$ has only $E_{G}(g)$-components, and $q^{\prime-1}$ has only $H_{\lambda}$-components. Thus the assumption (9) yields a contradiction. Hence, there exists $n \geq N$ such that for the element $a=g^{n} x$ we have $a \in H^{0}, \alpha(a) \in H^{0}$ and $a \stackrel{G}{\not} \alpha(a)$ 


\section{Commensurating automorphisms of Relatively hyperbolic groups}

The purpose of this section is to study automorphisms of relatively hyperbolic groups preserving commensurability classes.

Recall that $N_{G}(H)$ denotes the normalizer of a subgroup $H$ in a group $G$. Further, let $H$ be a non-elementary subgroup of a relatively hyperbolic group $G$ such that $H^{0} \neq \emptyset$. We denote by $\widehat{H}$ the product $H E_{G}(H)$. This is clearly a subgroup of G.

Theorem 5.1. Let $G$ be a relatively hyperbolic group, let $H \leq G$ be a nonelementary subgroup and let $\varphi \in \operatorname{Aut}(H)$. Suppose that $H^{0} \neq \emptyset$ and $\varphi(h) \stackrel{G}{\approx} h$ for every $h \in H^{0}$. Then there is a set map $\varepsilon: H \rightarrow E_{G}(H)$, whose restriction to $C_{H}\left(E_{G}(H)\right)$ is a homomorphism, and an element $w \in N_{G}(\widehat{H})$ such that for every $h \in H, \varphi(h)=w(h \varepsilon(h)) w^{-1}$.

Below is the main technical lemma of this section. It demonstrates how to construct the element $w$ and the restriction of the map $\varepsilon$ to $C_{H}\left(E_{G}(H)\right)$ from the statement of Theorem 5.1 .

Lemma 5.2. Suppose that $G$ is a relatively hyperbolic group, $H \leq G$ is a nonelementary subgroup and $\varphi \in \operatorname{Aut}(H)$. Assume that $H^{0} \neq \emptyset$ and $\varphi(h) \stackrel{G}{\approx} h$ for every $h \in H^{0}$. Then there is a homomorphism $\tilde{\varepsilon}: C_{H}\left(E_{G}(H)\right) \rightarrow E_{G}(H)$ and an element $w \in G$ such that for every $x \in C_{H}\left(E_{G}(H)\right), \varphi(x)=w(x \tilde{\varepsilon}(x)) w^{-1}$.

Proof. By Lemma 3.2, $H$ contains an $H$-special element $g_{1}$. Since $H$ is non-elementary and $C_{H}\left(E_{G}(H)\right)$ has finite index in it, $C_{H}\left(E_{G}(H)\right)$ is also non-elementary. The subgroup $E_{G}\left(g_{1}\right)$ is elementary (by part (1) of Lemma 2.4), thus there is an element $y \in C_{H}\left(E_{G}(H)\right) \backslash E_{G}\left(g_{1}\right)$. By Lemma 4.3. there is $k_{2} \in \mathbb{N}$ such that $g_{2}=g_{1}^{k_{2}} y \in C_{H}\left(E_{G}(H)\right)$ is loxodromic and $g_{2} \not \approx g_{1}$. Using the same lemma again we can find $k_{3} \in \mathbb{N}$ such that $g_{3}=g_{1}^{k_{3}} y \in C_{H}\left(E_{G}(H)\right)$ is loxodromic and $g_{3} \not{G} g_{i}$, $i=1,2$. In particular, $E_{G}\left(g_{2}\right) \cap\left\langle g_{3}\right\rangle=\{1\}$.

Choose $N_{4} \in \mathbb{N}$ according to an application of Lemma 4.5 to $\varphi, g_{1}, g_{2}, g_{3}$, and let $n_{3}=N_{4}$. By Lemma 4.3. there is $n_{2} \geq N_{4}$ such that $g_{2}^{n_{2}} g_{3}^{n_{3}} \in H^{0}$ is not commensurable with $g_{1}$ in $G$. Therefore $g_{2}^{n_{2}} g_{3}^{n_{3}} \in C_{H}\left(E_{G}(H)\right) \backslash E_{G}\left(g_{1}\right)$, and by Lemma 3.6 there is $N_{1} \in \mathbb{N}$ such that the element $g_{1}^{n} g_{2}^{n_{2}} g_{3}^{n_{3}}$ is $H$-special for any $n \geq N_{1}$. Denote $n_{1}=\max \left\{N_{1}, N_{4}\right\}$ and apply Lemma 4.3 to find $m \in \mathbb{N}$ such that the elements $a=g_{1}^{n_{1}} g_{2}^{n_{2}} g_{3}^{n_{3}}$ and $b=g_{1}^{n_{1}+m} g_{2}^{n_{2}} g_{3}^{n_{3}}$ are not commensurable with each other in $G$. In view of Lemma 4.5 one can conclude that the elements $a, b \in C_{H}\left(E_{G}(H)\right)$ are $H$-special and there exist $u, v \in G, \mu, \nu \in \mathbb{N}$ such that $\varphi\left(a^{\mu}\right)=u a^{\mu} u^{-1}, \varphi\left(b^{\nu}\right)=v b^{\nu} v^{-1}$.

Let $\chi: H \rightarrow G$ be the monomorphism, defined by $\chi(h)=u^{-1} \varphi(h) u$ for all

$h \in H$. Then $\chi\left(a^{\mu}\right)=a^{\mu}, \chi\left(b^{\nu}\right)=\left(u^{-1} v\right) b^{\nu}\left(u^{-1} v\right)^{-1}$. Note that $\chi(h) \stackrel{G}{\approx} h$ for every $h \in H^{0}$. By part (i) of Lemma 4.4, $a^{k \mu} b^{k \nu} \in H^{0}$ for every sufficiently large $k \in \mathbb{N}$. Therefore

$$
a^{k \mu}\left(u^{-1} v\right) b^{k \nu}\left(u^{-1} v\right)^{-1}=\chi\left(a^{k \mu} b^{k \nu}\right) \stackrel{G}{\approx} a^{k \mu} b^{k \nu} \text { for every sufficiently large } k \in \mathbb{N} .
$$

Consequently, by Lemma 4.6. $u^{-1} v \in E_{G}(a) E_{G}(b)$, thus $u^{-1} v=a^{s} b^{t} f$ for some $s, t \in \mathbb{Z}, f \in E_{G}(H)$. Hence $\chi\left(b^{\nu}\right)=a^{s} b^{\nu} a^{-s}$ because $b \in C_{H}\left(E_{G}(H)\right)$. Denote $w=u a^{s} \in G$ and let $\psi: H \rightarrow G$ be the monomorphism defined by the formula 
$\psi(h)=w^{-1} \varphi(h) w=a^{-s} \chi(h) a^{s}$ for all $h \in H$. By construction, we have

$$
\psi\left(a^{\mu}\right)=a^{\mu}, \psi\left(b^{\nu}\right)=b^{\nu} \text { and } \psi(h) \stackrel{G}{\approx} h \text { for each } h \in H^{0} .
$$

Choose any element $g \in S_{G}(H)$. We will show that there is $f \in E_{G}(H)$ such that $\psi(g)=g f$.

If $g \in E_{G}(a)$, then there is $n \in \mathbb{N}$ such that $g^{n} \in\left\langle a^{\mu}\right\rangle$ because $\left|E_{G}(a):\left\langle a^{\mu}\right\rangle\right|<$ $\infty$. Hence $\psi\left(g^{n}\right)=g^{n}$ and by Lemma 4.7 $\psi(g)=g f$ for some $f \in E_{G}(H)$.

Suppose, now, that $g \notin E_{G}(a)$. Since $g \in C_{H}\left(E_{G}(H)\right)$ and $a$ is $H$-special, we can use Lemmas 3.6 and 4.3 to find $l \in \mathbb{N}$ such that the element $d=a^{l \mu} g$ is $H$ special and is not commensurable with $a$ and $b$ in $G$. Arguing as in the beginning of the proof (using Lemmas 3.6, 4.3 and 4.5) we can find $m_{1}, m_{2}, m_{3} \in \mathbb{N}$ such that $c=a^{m_{1} \mu} b^{m_{2} \nu} d^{m_{3}} \in S_{G}(H), c \not{\sigma} a, c \not{\not} b$ and $\psi\left(c^{\zeta}\right)=e c^{\zeta} e^{-1}$ for some $\zeta \in \mathbb{N}$ and $e \in G$.

By part (i) of Lemma 4.4, $a^{k \mu} c^{k \zeta} \in H^{0}$ for every sufficiently large $k \in \mathbb{N}$. Hence $a^{k \mu} e c^{k \zeta} e^{-1}=\psi\left(a^{k \mu} c^{k \zeta}\right) \stackrel{G}{\approx} a^{k \mu} c^{k \zeta}$ whenever $k$ is sufficiently large. Applying Lemma 4.6 we see that $e \in E_{G}(a) E_{G}(c)$. As before, this implies that $\psi\left(c^{\zeta}\right)=$ $a^{p} c^{\zeta} a^{-p}$ for some $p \in \mathbb{Z}$.

Similarly, there is $q \in \mathbb{Z}$ such that $\psi\left(c^{\zeta}\right)=b^{q} c^{\zeta} b^{-q}$. Hence $\left(a^{-p} b^{q}\right) c^{\zeta}\left(a^{-p} b^{q}\right)^{-1}=$ $c^{\zeta}$, yielding that $a^{-p} b^{q} \in E_{G}(c)$.

Suppose that $p \neq 0$ and $q \neq 0$. Then the element $a^{-p} b^{q}$ must have infinite order (otherwise we would have $a^{-p} b^{q} \in E_{G}(H)$ since $c$ is $H$-special, hence $b^{q} \in$ $a^{p} E_{G}(H) \subset E_{G}(a)$, contradicting $\left.a \not ٌ b\right)$. This implies that $\left(a^{-p} b^{q}\right)^{\alpha}=c^{\beta}$ for some $\alpha \in \mathbb{Z} \backslash\{0\}$ and $\beta \in \mathbb{N}$. Recalling (10), we can apply Lemma 4.7 to find $f_{1}, f_{2} \in E_{G}(H)$ such that $\psi(a)=a f_{1}$ and $\psi(b)=b f_{2}$. Since $a, b \in C_{H}\left(E_{G}(H)\right)$ we obtain

$$
\psi\left(c^{\beta}\right)=\psi\left(\left(a^{-p} b^{q}\right)^{\alpha}\right)=\left(a^{-p} b^{q}\right)^{\alpha} f_{3}=c^{\beta} f_{3} \text { for some } f_{3} \in E_{G}(H) .
$$

Then for $\gamma=\beta \zeta\left|E_{G}(H)\right|$ we get $c^{\gamma}=\psi\left(c^{\gamma}\right)=a^{p} c^{\gamma} a^{-p}$, implying that $a^{p} \in E_{G}(c)$, which contradicts $a \not{G}$ c $c$.

Therefore either $p=0$ or $q=0$, thus $\psi\left(c^{\zeta}\right)=c^{\zeta}$. By Lemma 4.7 there is $f_{5} \in E_{G}(H)$ such that $\psi(c)=c f_{5}$. Since $c=a^{m_{1} \mu} b^{m_{2} \nu} d^{m_{3}}$, we can use (10) to get $\psi\left(d^{m_{3}}\right)=d^{m_{3}} f_{5}$. Applying Lemma 4.7 again, we find $f_{6} \in E_{G}(H)$ such that $\psi(d)=d f_{6}$. Finally, since $d=a^{l \mu} g$, in view of (10) we achieve $\psi(g)=g f_{6}$, as needed.

To finish the proof, we observe that by Proposition 3.3. $C_{H}\left(E_{G}(H)\right)$ is generated by $S_{G}(H)$, therefore for each $x \in C_{H}\left(E_{G}(H)\right)$ there is $\tilde{\varepsilon}(x) \in E_{G}(H)$ such that $\psi(x)=x \tilde{\varepsilon}(x)$. Since $\psi$ is a homomorphism, the map $\tilde{\varepsilon}: C_{H}\left(E_{G}(H)\right) \rightarrow E_{G}(H)$ will be a homomorphism too. By construction, we have $\varphi(x)=w \psi(x) w^{-1}=$ $w x \tilde{\varepsilon}(x) w^{-1}$.

Now we are ready to prove the main result of this section.

Proof of Theorem [5.1. Let $w \in G$ and $\tilde{\varepsilon}: C_{H}\left(E_{G}(H)\right) \rightarrow E_{G}(H)$ be as in the claim of Lemma 5.2, Let $\psi: H \rightarrow G$ be the monomorphism that is defined according to the formula $\psi(h)=w^{-1} \varphi(h) w$ for all $h \in H$. Denote $l=\left|H: C_{H}\left(E_{G}(H)\right)\right|$, $m=\left|E_{G}(H)\right|$ and $n=m l \in \mathbb{N}$.

Since $C_{H}\left(E_{G}(H)\right)$ is a normal subgroup of $H$, for any $z \in H$ we have $z^{l} \in$ $C_{H}\left(E_{G}(H)\right)$ and $\psi\left(z^{n}\right)=z^{n} \tilde{\varepsilon}\left(z^{l}\right)^{m}=z^{n}$. Fix an arbitrary $h \in H$. For any 
$g \in H^{0}$ we see that $g^{n}, h g^{n} h^{-1} \in C_{H}\left(E_{G}(H)\right) \cap H^{0}$, therefore $\psi(h) g^{n} \psi(h)^{-1}=$ $\psi\left(h g^{n} h^{-1}\right)=h g^{n} h^{-1}$, implying that $h^{-1} \psi(h) \in E_{G}(g)$. Thus, $h^{-1} \psi(h) \in$ $\bigcap_{g \in H^{0}} E_{G}(g)=E_{G}(H)$. After defining $\varepsilon(h)=h^{-1} \psi(h)$ for each $h \in H$, one immediately sees that $\varepsilon: H \rightarrow E_{G}(H)$ is a map with the required properties. Obviously, the restriction of $\varepsilon$ to $C_{H}\left(E_{G}(H)\right)$ coincides with $\tilde{\varepsilon}$.

It remains to prove that $w \in N_{G}(\widehat{H})$. We will first show that $w \in N_{G}\left(E_{G}(H)\right)$. Consider any element $f \in E_{G}(H)$. Since $\varphi$ is an automorphism of $H$, for any $g \in H^{0}$ there is $h \in H$ such that $\varphi(h)=g$. Then $h^{n} \in C_{H}\left(E_{G}(H)\right)$ and $g^{n}=$ $\varphi\left(h^{n}\right)=w h^{n} w^{-1}$ because $\varepsilon\left(h^{n}\right)=\tilde{\varepsilon}\left(h^{l}\right)^{m}=1$. Now we observe that

$$
w f w^{-1} g^{n}\left(w f w^{-1}\right)^{-1}=w f h^{n} f^{-1} w^{-1}=w h^{n} w^{-1}=g^{n} .
$$

Hence, $w f w^{-1} \in E_{G}(g)$ for every $g \in H^{0}$; consequently $w f w^{-1} \in E_{G}(H)$. The latter implies that $w E_{G}(H) w^{-1} \subseteq E_{G}(H)$ and since $E_{G}(H)$ is finite, we conclude that $w \in N_{G}\left(E_{G}(H)\right)$.

Now, for any $h \in H$ we have

$$
w h w^{-1}=w h \varepsilon(h) w^{-1} w \varepsilon(h)^{-1} w^{-1}=\varphi(h)\left(w \varepsilon(h) w^{-1}\right)^{-1} \in H E_{G}(H) ;
$$

thus $w H w^{-1} \subseteq \widehat{H}$. Since $w^{-1} \varphi(h) w=h \varepsilon(h) \in H E_{G}(H)$ and $\varphi \in A u t(H)$, one gets $w^{-1} H w \subseteq \widehat{H}$. Therefore $w \widehat{H} w^{-1} \subseteq \widehat{H} w E_{G}(H) w^{-1}=\widehat{H}, w^{-1} \widehat{H} w \subseteq$ $\widehat{H} w^{-1} E_{G}(H) w=\widehat{\widehat{H}}$, i.e., $w \in N_{G}(\widehat{H})$.

We are now in a position to prove Corollary 1.4 mentioned in the Introduction. We establish it in a more general form:

Corollary 5.3. Let $G$ be a non-elementary relatively hyperbolic group and $\varphi \in$ Aut $(G)$. The following conditions are equivalent:

(a) $\varphi$ is commensurating;

(b) $\varphi(g) \stackrel{G}{\approx} g$ for every loxodromic $g \in G$;

(c) there is a set map $\varepsilon: G \rightarrow E(G)$, whose restriction to $C(G)$ is a homomorphism, and an element $w \in G$ such that for every $g \in G, \varphi(g)=$ $w(g \varepsilon(g)) w^{-1}$.

In particular, if $E(G)=\{1\}$, then every commensurating automorphism of $G$ is inner.

Proof. (a) implies (b) by definition, and (b) implies (c) by Theorem 5.1. It remains to show that (c) implies (a). Indeed, let $g$ be an arbitrary element of $G$, and let the automorphism $\varphi$ satisfy (c). If $g$ is of finite order, then so is $\varphi(g)$, and in this case evidently $\varphi(g) \stackrel{G}{\approx} g$. Thus, we can suppose that $g$ has infinite order in $G$. By our assumptions, $\varphi(g)=w(g \varepsilon(g)) w^{-1}$ for some $w \in G$ and $\varepsilon(g) \in E(G)$. Since $E(G)$ is finite and normal in $G,\langle g\rangle$ has finite index in the subgroup $\langle g\rangle E(G)$. Hence there exists a non-zero integer $k$ such that $(g \varepsilon(g))^{k}=g^{l}$ for some $l \in \mathbb{Z}$, and since the order of $g \varepsilon(g)=w^{-1} \varphi(g) w$ is infinite, we can conclude that $l \neq 0$. Therefore $\varphi(g)=w g \varepsilon(g) w^{-1}$ is commensurable with $g$ in $G$. Thus $\varphi$ in commensurating.

Recall that a result of Metaftsis and Sykiotis [26, Lemma 2.2'] states that for any relatively hyperbolic group $G$, one has $\left|A u t_{c}(G): \operatorname{Inn}(G)\right|<\infty$, where

$$
\operatorname{Aut}_{c}(G)=\left\{\alpha \in \operatorname{Aut}(G) \mid \forall g \in G \exists x=x(g) \in G \text { such that } \alpha(g)=x g x^{-1}\right\}
$$


is the group of all pointwise inner automorphisms of $G$. Theorem 5.1 allows one to generalize their result to all non-elementary subgroups:

Corollary 5.4. Suppose that $H$ is a non-elementary subgroup of a relatively hyperbolic group $G$, with $H^{0} \neq \emptyset$. Then $\left|A u t_{c}(H): \operatorname{Inn}(H)\right|<\infty$. If, in addition, $E_{G}(H)=\{1\}$, then $\operatorname{Aut}_{c}(H)=\operatorname{Inn}(H)$.

Proof. By Theorem 5.1, for any automorphism $\varphi \in A u t_{c}(H)$, there exist $w \in G$ and a map $\varepsilon: H \rightarrow E_{G}(H)$ such that $\varphi(h)=w h \varepsilon(h) w^{-1}$ for each $h \in H$. Take any element $h \in S_{G}(H)$. Then $h$ commutes with $\varepsilon(h) \in E_{G}(H)$, and, consequently, $(\varphi(h))^{n}=w h^{n} w^{-1}$, where $n=\left|E_{G}(H)\right| \in \mathbb{N}$.

Now, since $\varphi$ is a pointwise inner automorphism of $H$, there is $x \in H$ such that $\varphi(h)=x h x^{-1}$. Hence $x h^{n} x^{-1}=w h^{n} w^{-1}$, i.e., $w^{-1} x \in E_{G}(h)=\langle h\rangle \times E_{G}(H)$. Thus $w=f z$ for some $f \in H$ and $z \in E_{G}(H)$, and $w^{-1} x \in C_{G}(h)$ because $h$ is $H$-special. Consequently, we have $h=w^{-1} x h\left(w^{-1} x\right)^{-1}=h \varepsilon(h)$, which implies that $\varepsilon(h)=1$. Since the latter holds for any $h \in S_{G}(H)$, it follows from Proposition 3.3 that $\varepsilon\left(C_{H}\right)=\{1\}$, where $C_{H}=C_{H}\left(E_{G}(H)\right)$.

Note that $\left|H: C_{H}\right|<\infty$, hence there are $h_{1}, \ldots, h_{l} \in H$ such that $H=$ $\bigsqcup_{i=1}^{l} C_{H} h_{i}$. For any $g \in H$ there are $a \in C_{H}$ and $i \in\{1, \ldots, l\}$ such that $g=a h_{i}$. One has

$$
\begin{aligned}
\varphi(a) \varphi\left(h_{i}\right)=\varphi(g)=w g \varepsilon(g) w^{-1} & =w a w^{-1} w h_{i} \varepsilon\left(a h_{i}\right) w^{-1} \\
& =\varphi(a) \varphi\left(h_{i}\right) w\left(\varepsilon\left(h_{i}\right)\right)^{-1} \varepsilon\left(a h_{i}\right) w^{-1}
\end{aligned}
$$

Hence $\varepsilon(g)=\varepsilon\left(a h_{i}\right)=\varepsilon\left(h_{i}\right)$; i.e., the map $\varepsilon$ is uniquely determined by the images of $h_{1}, \ldots, h_{l}$. Thus, $\varphi(g)=f z\left(g \varepsilon\left(h_{i}\right)\right) z^{-1} f^{-1}$, implying that the automorphism $\varphi \in \operatorname{Aut}_{c}(H)$, up to composition with an inner automorphism of $H$, is completely determined by the finite collection of elements $z, \varepsilon\left(h_{1}\right), \ldots, \varepsilon\left(h_{l}\right) \in E_{G}(H)$, and since $E_{G}(H)$ is finite, we can conclude that $\left|A u t_{c}(H): \operatorname{Inn}(H)\right|<\infty$.

Now, if $E_{G}(H)=\{1\}$ we obtain $w=f \in H$ and $\varphi(g)=w g w^{-1}$ for all $g \in H$, that is, $\varphi \in \operatorname{Inn}(H)$.

\section{Group-theoretic Dehn surgery and normal automorphisms}

In the context of relatively hyperbolic groups, the algebraic analogue of Dehn filling is defined as follows. Suppose that $\left\{H_{\lambda}\right\}_{\lambda \in \Lambda}$ is a collection of (peripheral) subgroups of a group $G$. To each collection $\mathfrak{N}=\left\{N_{\lambda}\right\}_{\lambda \in \Lambda}$, where $N_{\lambda}$ is a normal subgroup of $H_{\lambda}$, we associate the quotient-group

$$
G(\mathfrak{N})=G /\left\langle\left\langle\bigcup_{\lambda \in \Lambda} N_{\lambda}\right\rangle\right\rangle^{G}
$$

Definition 6.1. Let $G$ and $\left\{H_{\lambda}\right\}_{\lambda \in \Lambda}$ be as described above. We say that some assertion holds for most peripheral fillings of $G$ if there exists a finite subset $\mathcal{F}$ of non-trivial elements of $G$ such that the assertion holds for $G(\mathfrak{N})$ for any collection $\mathfrak{N}=\left\{N_{\lambda}\right\}_{\lambda \in \Lambda}$ of normal subgroups $N_{\lambda} \triangleleft H_{\lambda}$ satisfying $N_{\lambda} \cap \mathcal{F}=\emptyset$ for all $\lambda \in \Lambda$.

The theorem below was proved in 32 . In the particular case when $G$ is torsionfree, this theorem was independently proved in [18, 19].

Theorem 6.2. Suppose that a group $G$ is hyperbolic relative to a collection of subgroups $\left\{H_{\lambda}\right\}_{\lambda \in \Lambda}$. Then for most peripheral fillings of $G$, the following holds.

1) For each $\lambda \in \Lambda$, the natural map $H_{\lambda} / N_{\lambda} \rightarrow G(\mathfrak{N})$ is injective. 
2) The quotient group $G(\mathfrak{N})$ is hyperbolic relative to the collection $\left\{H_{\lambda} / N_{\lambda}\right\}_{\lambda \in \Lambda}$.

The following statement plays a key role in our paper.

Lemma 6.3. Let $G$ be a relatively hyperbolic group, $H$ a subgroup of $G$ and $\alpha \in$ Aut $(H)$. Suppose that there exists a loxodromic element $g \in H$ such that $\alpha(g)$ is not conjugate to an element of $E_{G}(g)$ in $G$. Then $\alpha$ does not preserve some normal subgroup of $H$.

Proof. Suppose that $G$ is hyperbolic relative to $\left\{H_{\lambda}\right\}_{\lambda \in \Lambda}$. There are two cases to consider.

Case 1. Assume first that $\alpha(g)$ is loxodromic. Using Lemma 2.4 twice we obtain that $G$ is hyperbolic relative to $\left\{H_{\lambda}\right\}_{\lambda \in \Lambda} \cup\left\{E_{G}(g), E_{G}(\alpha(g))\right\}$. Since $\langle g\rangle$ has finite index in $E_{G}(g)$, there is $m \neq 0$ such that $\left\langle g^{m}\right\rangle$ (and each of its subgroups) is normal in $E_{G}(g)$. Let $\mathcal{F}$ be the finite set provided by Theorem 6.2 for the peripheral system $\left\{H_{\lambda}\right\}_{\lambda \in \Lambda} \cup\left\{E_{G}(g), E_{G}(\alpha(g))\right\}$. Taking $p$ to be a sufficiently large multiple of $m$, we can ensure the condition $\left\langle g^{p}\right\rangle \cap \mathcal{F}=\emptyset$. We now consider the filling of $G$ with respect to the collection of subgroups $\mathfrak{N}$ consisting of the trivial subgroups of $H_{\lambda}$ 's, the trivial subgroup of $E_{G}(\alpha(g))$, and $\left\langle g^{p}\right\rangle \triangleleft E_{G}(g)$. By Theorem 6.2, elements $g$ and $\alpha(g)$ have orders $p$ and $\infty$, respectively, in $Q=G /\left\langle\left\langle g^{p}\right\rangle\right\rangle^{G}$. Hence $\alpha$ does not induce an automorphism on the natural image of $H$ in $Q$; i.e., it does not preserve $\left\langle\left\langle g^{p}\right\rangle\right\rangle^{G} \cap H$.

Case 2. Now suppose that $\alpha(g)$ is parabolic; i.e., it is conjugate to an element of some peripheral subgroup $H_{\lambda}$. Again, by Lemma 2.4. $G$ is hyperbolic relative to $\left\{H_{\lambda}\right\}_{\lambda \in \Lambda} \cup\left\{E_{G}(g)\right\}$. The rest of the proof is identical to that in Case 1. The only difference is that Theorem 6.2 is applied to the collection of subgroups $\mathfrak{N}$ consisting of trivial subgroups of $H_{\lambda}$ 's and $\left\langle g^{p}\right\rangle \triangleleft E_{G}(g)$ for some $p>0$.

Theorem 1.1 is a particular case of the following result. (Recall that $\widehat{H}=$ $H E_{G}(H)$.)

Theorem 6.4. Let $G$ be a relatively hyperbolic group and let $H \leq G$ be a nonelementary subgroup such that $H^{0} \neq \emptyset$. Then for any $\varphi \in A_{u} t_{n}(H)$ there exists a map $\varepsilon: H \rightarrow E_{G}(H)$, whose restriction to $C_{H}\left(E_{G}(H)\right)$ is trivial, and an element $w \in N_{G}(\widehat{H})$ such that for every $h \in H, \varphi(h)=w h \varepsilon(h) w^{-1}$.

Proof. By Lemma 6.3 $\varphi$ maps every loxodromic element $h \in H$ to a conjugate of an element of $E_{G}(h)$. As $\langle h\rangle$ has finite index in $E_{G}(h)$, every element of infinite order in $E_{G}(h)$ is commensurable with $h$ in $G$. In particular, $\varphi(h) \stackrel{G}{\approx} h$ for every $h \in H^{0}$. Hence by Theorem 5.1, there is a map $\varepsilon: H \rightarrow E_{G}(H)$, whose restriction to $C_{H}\left(E_{G}(H)\right)$ is a homomorphism, and an element $w \in N_{G}(\widehat{H})$ such that for every $h \in H, \varphi(h)=w h \varepsilon(h) w^{-1}$. It remains to show that $\varepsilon(h)=1$ for every $h \in C_{H}\left(E_{G}(H)\right)$.

By Proposition 3.3, it suffices to show that $\varepsilon(h)=1$ for all $h \in S_{G}(H)$. Suppose that $\varphi(h)=w h r w^{-1}$ for some $r \in E_{G}(H) \backslash\{1\}$. Take any integer $p \equiv 1(\bmod |r|)$, where $|r|$ denotes the (finite) order of $r$ in $G$. Note that $h$ commutes with $r$ as $h \in S_{G}(H)$. Thus $\varphi\left(h^{p}\right)=w h^{p} r w^{-1}$. Since $\varphi$ should preserve $\left\langle\left\langle h^{p}\right\rangle\right\rangle^{G} \cap H$, we obtain $h^{p} r \in\left\langle\left\langle h^{p}\right\rangle\right\rangle^{G}$. On the other hand, $h^{p} r \in E_{G}(h)$. By Lemma 2.4 we can join $E_{G}(h)$ to the collection of the peripheral subgroups. Without loss of generality 
we may assume that $p \gg 1$ so that the normal subgroup $N=\left\langle h^{p}\right\rangle$ of $E_{G}(h)$ satisfies the requirement $N \cap \mathcal{F}=\emptyset$ from Theorem 6.2 (and Definition 6.1). Then by the first part of Theorem 6.2 we have $h^{p} r \in\left\langle\left\langle h^{p}\right\rangle\right\rangle^{G} \cap E_{G}(h)=\left\langle h^{p}\right\rangle$. Hence $r \in\langle h\rangle \cap E_{G}(H)=\{1\}$, which contradicts $r \neq 1$.

Corollary 6.5. Let $H$ be a non-elementary subgroup of a relatively hyperbolic group $G$ such that $H^{0} \neq \emptyset$. Then the following hold.

a) If $H$ has finite index in $N_{G}\left(H E_{G}(H)\right)$, then $O u t_{n}(H)$ is finite.

b) If $H$ does not normalize any non-trivial finite subgroup of $G$, and $H=$ $N_{G}(H)$, then $\mathrm{Out}_{n}(H)=\{1\}$.

Proof. The argument is similar to the one used to prove Corollary 5.4. Observe that by Lemma 2.5, $E_{G}(H)$ is a finite subgroup of $G$ normalized by $H$. Therefore $H$ acts on $E_{G}(H)$ by conjugation, and $C_{H}=C_{H}\left(E_{G}(H)\right)$ has a finite index in $H$ as a kernel of this action.

Let $h_{1}, \ldots, h_{l}$ be elements of $H$ such that $H=\bigsqcup_{i=1}^{l} C_{H} h_{i}$. By Theorem 6.4 we can argue as in the proof of Corollary 5.4 to conclude that every normal automorphism $\varphi$ of $H$ is uniquely determined by the images $\varepsilon\left(h_{i}\right)$ of $h_{i}, i=1, \ldots, l$, and by the conjugating element $w \in N_{G}(\widehat{H})$. As $E_{G}(H)$ is finite, for each $i$ there are only finitely many possibilities for $\varepsilon\left(h_{i}\right)$, and since $\left|N_{G}(\widehat{H}): H\right|<\infty$, we can deduce that $\left|\operatorname{Aut}_{n}(H): \operatorname{Inn}(H)\right|<\infty$.

Furthermore, if $H=N_{G}(H)$ and $H$ does not normalize any finite normal subgroup of $G$, we obtain $E_{G}(H)=\{1\}, N_{G}(\widehat{H})=N_{G}(H)=H$, and $C_{H}\left(E_{G}(H)\right)=$ $H$. Hence $\operatorname{Aut}_{n}(H)=\operatorname{Inn}(H)$ by Theorem 6.4, This completes the proof.

The next lemma shows that Corollary 1.2 holds for elementary groups.

Lemma 6.6. Let $G$ be a virtually cyclic group. Then Out $(G)$ is finite.

Proof. If $G$ is finite the claim is trivial, so assume that $G$ is infinite. Recall that every elementary group is ether finite-by-cyclic or finite-by-(infinite dihedral) (see, for example, [15, Lemma 2.5]). More precisely, as $G$ is infinite, the quotient $G / E(G)$ (where $E(G)$ is the maximal finite normal subgroup of $G$ given by Corollary 2.6) is either infinite cyclic or infinite dihedral. In both cases we have

$$
|A u t(G / E(G)): \operatorname{Inn}(G / E(G))|=2 .
$$

Every automorphism $\alpha \in \operatorname{Aut}(G)$ induces an automorphism $\bar{\alpha} \in \operatorname{Aut}(G / E(G))$. This gives rise to a homomorphism $\xi: \operatorname{Aut}(G) \rightarrow \operatorname{Aut}(G / E(G))$. If $\alpha \in \operatorname{ker}(\xi)$, then for every $x \in G$ there is $h=h(x) \in E(G)$ such that $\alpha(x)=x h$. By our assumptions, $G$ is generated by a finite set of elements $\left\{x_{i} \mid i=1, \ldots, n\right\}$ and the automorphism $\alpha$ is uniquely determined by the images $\alpha\left(x_{i}\right), i=1, \ldots, n$. Since $|E(G)|<\infty$, for each $i$ there are only finitely many possibilities for $h\left(x_{i}\right)$. Therefore the kernel of $\xi$ is finite. Evidently $\xi(\operatorname{Inn}(G))=\operatorname{Inn}(G / E(G))$, and by (12) we get $|\operatorname{Aut}(G):(\operatorname{Inn}(G) \operatorname{ker}(\xi))| \leq 2$ yielding that $\mid$ Out $(G)|=| \operatorname{Aut}(G): \operatorname{Inn}(G) \mid<$ $\infty$.

Proof of Theorem 1.1. Let us apply Theorem 6.4 to the case $G=H$. Then $E_{G}(H)$ $=E(G), C_{H}\left(E_{G}(H)\right)=C(G), \widehat{H}=N_{G}(\widehat{H})=G$, and the claim of Theorem 1.1 follows immediately. 
Proof of Corollary 1.2. First, suppose that $G$ is elementary. In this case the first part of the corollary follows from Lemma 6.6. To derive the second claim of the corollary, we observe that since $G$ is non-cyclic and does not have non-trivial finite normal subgroups, it must be infinite dihedral (this follows from the structure of an elementary group; see the proof of Lemma 6.6). Hence $G \cong \mathbb{Z} / 2 \mathbb{Z} * \mathbb{Z} / 2 \mathbb{Z}$ and, by Neshchadim's theorem [29], Out ${ }_{n}(G)=\{1\}$.

Thus we may assume that $G$ is non-elementary. In this case the corollary follows from Theorem 1.1 in the same way that Corollary 6.5 follows from Theorem 6.4 . Alternatively it follows immediately from Corollary 6.5 applied to the case when $G=H$.

\section{FREE PRODUCTS AND GROUPS WITH INFINITELY MANY ENDS}

In order to prove Theorem 1.6 we need two more statements below.

Lemma 7.1. Assume that $G$ is a relatively hyperbolic group and $g, h$ are two noncommensurable loxodromic elements. Then $g$ and $h$ are non-commensurable and loxodromic in most peripheral fillings of $G$.

Proof. Suppose that $G$ is hyperbolic relative to a collection of subgroups $\left\{H_{\lambda}\right\}_{\lambda \in \Lambda}$. Applying Lemma 2.4 twice we obtain that $G$ is hyperbolic relative to the new collection $\left\{H_{\lambda}\right\}_{\lambda \in \Lambda} \cup\left\{E_{1}, E_{2}\right\}$, where $E_{1}=E_{G}(g), E_{2}=E_{G}(h)$. Let $\mathcal{F}_{1}$ and $\mathcal{F}_{2}$ be the finite subsets provided by Theorem 6.2 for the collections of peripheral subgroups $\left\{H_{\lambda}\right\}_{\lambda \in \Lambda}$ and $\left\{H_{\lambda}\right\}_{\lambda \in \Lambda} \cup\left\{E_{1}, E_{2}\right\}$, respectively. Set $\mathcal{F}=\mathcal{F}_{1} \cup \mathcal{F}_{2}$.

Consider any collection of subgroups $N_{\lambda} \triangleleft H_{\lambda}$ such that $N_{\lambda} \cap \mathcal{F}=\emptyset, \lambda \in \Lambda$. By Theorem 6.2 the filling of $G$ with respect to the collection of normal subgroups $\mathfrak{N}$, consisting of $N_{\lambda} \triangleleft H_{\lambda}$ for $\lambda \in \Lambda$ and the trivial subgroups of $E_{1}, E_{2}$, is hyperbolic relative to $\left\{H_{\lambda} / N_{\lambda}\right\}_{\lambda \in \Lambda} \cup\left\{E_{1}, E_{2}\right\}$ as well as relative to $\left\{H_{\lambda} / N_{\lambda}\right\}_{\lambda \in \Lambda}$. (We keep the same notation for the isomorphic images of $E_{1}, E_{2}$ in $G(\mathfrak{N})$ and the elements $g, h$.

In particular, $E_{1} \cap E_{2}^{t}$ is finite for every $t \in G(\mathfrak{N})$. Clearly this implies that $g$ and $h$ are not commensurable in $G$. Similarly $g$ and $h$ are not conjugate to any elements of the subgroups $H_{\lambda} / N_{\lambda}, \lambda \in \Lambda$, of $G(\mathfrak{N})$. Thus $g$ and $h$ are loxodromic in $G(\mathfrak{N})$ with respect to the peripheral collection $\left\{H_{\lambda} / N_{\lambda}\right\}_{\lambda \in \Lambda}$. As $\mathcal{F}$ is finite, $g$ and $h$ are non-commensurable and loxodromic in most peripheral fillings of $G$ (with respect to the peripheral structure $\left.\left\{H_{\lambda}\right\}_{\lambda \in \Lambda}\right)$.

The proof of Theorem 1.6 uses the following lemma, which is an immediate corollary of 45. Lemma 3]. (Recall that the Cartesian subgroup of a free product $A * B$ is, by definition, the kernel of the natural epimorphism $A * B \rightarrow A \times B$.)

Lemma 7.2. Let $G=A * B$, where $A$ and $B$ are finite groups. Let $u$, $v$ be noncommensurable elements of the Cartesian subgroup $C$ of $G$. Suppose that $u=a^{k}$, $v=b^{l}$ for some positive integers $k, l$, where $a, b$ are not proper powers. Assume also that $a^{k}$ (respectively, $b^{l}$ ) is the smallest non-zero power of a (respectively, $b$ ) that belongs to $C$. Then there exists a finite quotient-group $Q$ of $G$ such that the images of $u$ and $v$ have different orders in $Q$.

Proof of Theorem 1.6. Let $G$ be a non-trivial free product, i.e., $G=A * B$, where both $A$ and $B$ are non-trivial. Then $G$ is hyperbolic with respect to $\{A, B\}$ (the finite sets $X$ and $\mathcal{R}$, from the definition of relative hyperbolicity in Section 2, can be taken to be empty; the isoperimetric constant $C$ for the corresponding relative 
presentation of $G$ will then be equal to zero). In what follows, we will fix this as a system of peripheral subgroups of $G$.

If $|A|=|B|=2$, the proof is an easy exercise. It also follows from the main result of [29], stating that every normal automorphism of a non-trivial free product is inner, and the observation that every non-trivial normal subgroup of the infinite dihedral group is of finite index.

Thus we may assume that $G$ is non-elementary. Suppose that there exists an automorphism $\alpha \in \operatorname{Aut}_{n}^{f}(G) \backslash \operatorname{Inn}(G)$. Note that $E(G)=\{1\}$ because $G$, as a non-trivial free product, cannot contain non-trivial finite normal subgroups. Since $\alpha$ is not an inner automorphism of $G$, it follows from Corollary 1.4 that $\alpha$ is not commensurating. Therefore, by Corollary 5.3 and Lemma 4.8 (applied to the case when $H=G)$, there is a loxodromic element $g \in G$ such that $h=\alpha(g)$ is also loxodromic and is not commensurable with $g$. Further, by Lemma 7.1 there exist finite index normal subgroups $M \triangleleft A$ and $N \triangleleft B$ such that the natural images $\bar{g}$, $\bar{h}$ of $g$ and $h$, respectively, are not commensurable in $\bar{G}=A / M * B / N$. Without loss of generality we may assume that $\bar{G}$ is non-elementary.

Since $\bar{G}$ is a free product of two finite groups, it is residually finite. Therefore the kernel $K$ of the natural homomorphism $G \rightarrow \bar{G}$ is an intersection of finite index normal subgroups of $G$. As $\alpha \in A u t_{n}^{f}(G), \alpha$ stabilizes $K$. Hence $\alpha$ induces an automorphism $\bar{\alpha}$ of $\bar{G}$.

Let $\bar{g}=a^{k}$, where $k$ is a positive integer and $a$ is not a proper power. Clearly $b=\bar{\alpha}(a)$ is not a proper power as well and $b^{k}=\bar{h}$. Evidently $b^{p}=\bar{\alpha}\left(a^{p}\right)$ is not commensurable to $a^{p}$ for any non-zero integer $p$. Let $C$ denote the Cartesian subgroup of $\bar{G}$. Then $|\bar{G}: C|<\infty$, and replacing $\bar{g}$ with another positive power of $a$, if necessary, we may assume that $k>0$ and that $\bar{g}=a^{k}$ is the smallest nonzero power of $a$ that belongs to $C$. Again, since $|\bar{G}: C|<\infty, \bar{\alpha}$ preserves $C$. In particular, $\bar{h}=b^{k}$ is the smallest power of $b$ that belongs to $C$.

By Lemma 7.2 there exists a finite index normal subgroup $K$ of $\bar{G}$ such that the images of $\bar{g}$ and $\bar{h}$ have different orders in $\bar{G} / K$. Therefore $\bar{\alpha}$ does not induce an automorphism on $\bar{G} / K$. Obviously this means that $\alpha$ does not preserve the full preimage of $K$ in $G$, which contradicts our assumption that $\alpha \in A u t_{n}^{f}(G)$.

The following lemma is well known and is easy to prove (see, for example, 21, Lemma 5.4]).

Lemma 7.3. Suppose that $G$ is a finitely generated group and $N$ is a centerless normal subgroup of finite index in $G$. Then some finite index subgroup of Out $(G)$ is isomorphic to a quotient of a subgroup of Out $(N)$ by a finite normal subgroup. In particular, if Out $(N)$ is residually finite, then $\operatorname{Out}(G)$ is residually finite.

The next observation is trivial.

Lemma 7.4. Suppose that a group $G$ acts on a set $\mathcal{M}$ faithfully with finite orbits. Then $G$ is residually finite.

Proof. Given $g \in G$, let $s \in \mathcal{M}$ be an element such that $g(s) \neq s$. Then the natural map from $G$ to the symmetric group on the orbit of $s$ provides us with a finite quotient of $G$, where the image of $g$ is non-trivial.

Proof of Theorem 1.5. By Stallings's Theorem (41, 42]) there is a finite group $S$ such that $G$ splits as an amalgamated free product $A *_{S} B$ or an $H N N$-extension $A *_{S}$, where $(|A: S|-1)(|B: S|-1) \geq 2$ in the first case and $\left|A: S_{i}\right| \geq 2, i=1,2$, 
in the second case (where $S_{1}$ and $S_{2}$ are the two associated isomorphic copies of $S$ in $A$ ). Since $G$ is residually finite and $S$ is finite, there exists a finite index normal subgroup $N \triangleleft G$ such that $N \cap S=\{1\}$ if $G=A *_{S} B$, or $N \cap S_{i}=\{1\}$ for $i=1,2$, if $G=A *_{S}$. Note that the quotient of the Bass-Serre tree for $G$ modulo the action of $N$ is finite and the edge stabilizers in $N$ are trivial. The Bass-Serre structure theorem for groups acting on trees (see [39]) yields a splitting of $N$ into a non-trivial free product. In particular, $N$ is centerless.

The group $\operatorname{Aut}(N)$ naturally acts on the set $\mathcal{M}$ of finite index normal subgroups of $N$ and $A u t_{n}^{f}(N)$ is the kernel of this action. By Theorem 1.6. $A u t_{n}^{f}(N)=$ $\operatorname{Inn}(N)$. Therefore, $\operatorname{Aut}(N) / \operatorname{Aut}_{n}^{f}(N)=\operatorname{Aut}(N) / \operatorname{Inn}(N)=\operatorname{Out}(N)$ acts on $\mathcal{M}$ faithfully. Since $N$ is finitely generated, there are only finitely many subgroups of a given finite index in $N$, thus all orbits of the action of $\operatorname{Out}(N)$ on $\mathcal{M}$ are finite. Hence $\operatorname{Out}(N)$ is residually finite by Lemma 7.4. The claim of the theorem is now a consequence of Lemma 7.3 ,

\section{ACKNOWLEDGMENTS}

We are grateful to A. Klyachko and V. Yedynak for useful discussions, and to the anonymous referee for his comments.

\section{REFERENCES}

[1] R.B.J.T. Allenby, G. Kim, C.Y. Tang, On the residual finiteness of $\operatorname{Out}\left(\pi_{1}(M)\right)$ of certain Seifert manifolds, Algebra Colloq. 10 (2003), no. 2, 121-126. MR.1980432 (2004d:20030)

[2] R.B.J.T. Allenby, G. Kim, C.Y. Tang, Residual finiteness of outer automorphism groups of certain pinched 1-relator groups, J. Algebra 246 (2001), no. 2, 849-858. MR1872127 (2002i:20052)

[3] G. Arzhantseva, A. Minasyan, D. Osin, The SQ-universality and residual properties of relatively hyperbolic groups, J. Algebra 315 (2007), no. 1, 165-177. MR2344339 (2008g:20095)

[4] G. Baumslag, Automorphism groups of residually finite groups, J. London Math. Soc. 38 (1963), 117-118. MR0146271 (26:3793)

[5] M. Bestvina, Degenerations of the hyperbolic space, Duke Math. J. (1) 56 (1988), 143-161. MR 932860 (89m:57011)

[6] O. Bogopolski, E. Kudryavtseva, H. Zieschang, Simple curves on surfaces and an analog of a theorem of Magnus for surface groups, Math. Z. 247 (2004), no. 3, 595-609. MR 2114430 (2005k:20087)

[7] B.H. Bowditch, Relatively hyperbolic groups, preprint, 1999.

[8] I. Bumagin, D. Wise, Every group is an outer automorphism group of a finitely generated group, J. Pure Appl. Algebra 200 (2005), 137-147. MR2142354(2005m:20085)

[9] E.A. Cherepanov, Normal automorphisms of free Burnside groups of large odd exponents, Internat. J. Algebra Comput. 16 (2006), no. 5, 839-847. MR2274717(2007h:20025)

[10] F. Dahmani, Combination of convergence groups, Geom. \& Topol. 7 (2003), 933-963. MR2026551 (2005g:20063)

[11] C. Druţu, M. Sapir, Tree-graded spaces and asymptotic cones of groups. With an appendix by D. Osin and M. Sapir. Topology 44 (2005), no. 5, 959-1058. MR2153979 (2006d:20078)

[12] M. Droste, M. Giraudet, R. Göbel, All groups are outer automorphism groups of simple groups, J. London Math. Soc. (2) 64 (2001), no. 3, 565-575. MR.1865550 (2002h:20050)

[13] G. Endimioni, Pointwise inner automorphisms in a free nilpotent group, Q. J. Math. 53 (2002), no. 4, 397-402. MR 1949151(2003i:20047)

[14] B. Farb, Relatively hyperbolic groups, Geom. Funct. Anal. 8 (1998), 810-840. MR.1650094 (99j:20043)

[15] F.T. Farrell, L.E. Jones, The lower algebraic $K$-theory of virtually infinite cyclic groups, K-Theory 9 (1995), 13-30. MR1340838(96e:19003) 
[16] M. Gromov, Hyperbolic groups, Essays in Group Theory, MSRI Series, Vol.8 (S.M. Gersten, ed.), Springer, 1987, 75-263. MR919829 (89e:20070)

[17] E. Grossman, On the residual finiteness of certain mapping class groups, J. London Math. Soc. 9 (1974/75), no. 2, 160-164. MR0405423 (53:9216)

[18] D. Groves, J. Manning, Dehn filling in relatively hyperbolic groups, Israel J. Math. 168 (2008), 317-429. MR2448064

[19] D. Groves, J. Manning, Fillings, finite generation, and direct limits of relatively hyperbolic groups, Groups Geom. Dyn. 1 (2007), no. 3, 329-342. MR2314049(2008f:20106)

[20] V. Guirardel, Limit groups and groups acting freely on $\mathbb{R}^{n}$-trees, Geom. Topol. 8 (2004), 1427-1470. MR 2119301 (2005m:20060)

[21] V. Guirardel, G. Levitt, The outer space of a free product, Proc. London Math. Soc. 94 (2007), no. 3, 695-714. MR2325317 (2008c:20047)

[22] C. Hrushka, Relative hyperbolicity and relative quasiconvexity for countable groups, preprint. arXiv:0801.4596.

[23] C. Hruska, B. Kleiner, Hadamard spaces with isolated flats (with an appendix by the authors and Mohamad Hindawi), Geom. Topol. 9 (2005), 1501-1538. MR2175151 (2007c:20105)

[24] O. Kharlampovich, A. Myasnikov, Elementary theory of free non-abelian groups, J. Algebra 302 (2006), no. 2, 451-552. MR2293770(2008e:20033)

[25] A. Lubotzky, Normal automorphisms of free groups, J. Algebra 63 (1980), no. 2, 494-498. MR.570726 (81k:20041)

[26] V. Metaftsis, M. Sykiotis, On the residual finiteness of outer automorphisms of relatively hyperbolic groups, preprint. arXiv:math/0608685v2.

[27] A. Minasyan, Groups with finitely many conjugacy classes and their automorphisms, Comm. Math. Helv. 84 (2009), no. 2, 259-296. MR2495795 (2010b:20052)

[28] A. Minasyan, On residualizing homomorphisms preserving quasiconvexity, Comm. in Algebra 33 (2005), no. 7, 2423-2463. MR2153233 (2006c:20089)

[29] M.V. Neshchadim, Free products of groups that do not have outer normal automorphisms, Algebra and Logic 35 (1996), no. 5, 316-318. MR1444437 (98c:20048)

[30] V.N. Obraztsov, Embedding into groups with well-described lattices of subgroups, Bull. Austral. Math. Soc. 54 (1996), no. 2, 221-240. MR1411532(97k:20051)

[31] D.V. Osin, Elementary subgroups of relatively hyperbolic groups and bounded generation, Internat. J. Algebra Comput. 16 (2006), no. 1, 99-118. MR2217644 (2007b:20092)

[32] D.V. Osin, Peripheral fillings of relatively hyperbolic groups, Invent. Math. 167 (2007), no. 2, 295-326. MR 2270456 (2008d:20080)

[33] D.V. Osin, Relatively hyperbolic groups: Intrinsic geometry, algebraic properties, and algorithmic problems, Mem. Amer. Math. Soc. 179 (2006), no. 843. MR2182268 (2006i:20047)

[34] F. Paulin, Outer automorphisms of hyperbolic groups and small actions on $\mathbb{R}$-trees. In: Arboreal group theory (Berkeley, CA), 331-343. Math. Sci. Res. Inst. Publ. 19, Springer, New York, 1991. MR1105339 (92g:57003)

[35] D.Y. Rebbechi, Algorithmic properties of relatively hyperbolic groups, Ph.D. thesis. arXiv: math/0302245.

[36] V.N. Remeslennikov, Finite approximability of groups with respect to conjugacy. (Russian) Siberian Math. J. 23 (1971), 783-792. MR0294469 (45:3539)

[37] V.A. Roman'kov, Normal automorphisms of discrete groups (in Russian), Sibirsk. Mat. Zh. 24 (1983), no. 4, 138-149. MR713591 (86k:20027)

[38] C. Sah, Automorphisms of finite groups, J. Algebra 10 (1968), 47-68. MR0229713 (37:5287)

[39] J.-P. Serre, Arbres, amalgames, $\mathrm{SL}_{2}$, Astérisque, no. 46. Société Mathématique de France, Paris, 1977. 189 pp. MR0476875 (57:16426)

[40] Z. Sela, Diophantine geometry over groups. VI. The elementary theory of a free group, Geom. Funct. Anal. 16 (2006), no. 3, 707-730. MR.2238945 (2007j:20063)

[41] J. Stallings, Group theory and three-dimensional manifolds, Yale Mathematical Monographs, 4. Yale University Press, New Haven, Conn.-London, 1971. MR0415622 (54:3705)

[42] J. Stallings, On torsion-free groups with infinitely many ends, Ann. of Math. (2) 88 (1968), 312-334. MR0228573 (37:4153)

[43] P. Tukia, Convergence groups and Gromov's metric hyperbolic spaces, New Zeland J. Math 23 (1994), 157-187. MR1313451 (96c:30042) 
[44] A. Yaman, A topological characterization of relatively hyperbolic groups, J. Reine Angew. Math. 566 (2004), 41-89. MR2039323 (2005e:20064)

[45] V. Yedynak, Multielement order separability in free products of groups, Comm. in Algebra, to appear.

School of Mathematics, University of Southampton, Highfield, Southampton, SO17 1BJ, United Kingdom.

E-mail address: aminasyan@gmail.com

Department of Mathematics, Vanderbilt University, Nashville, Tennessee 37240

E-mail address: denis.osin@gmail.com 